\title{
UNIFORM AUXILIARY SPACE PRECONDITIONING FOR HDG METHODS FOR ELLIPTIC OPERATORS WITH A PARAMETER DEPENDENT LOW ORDER TERM
}

\author{
GUOSHENG FU
}

\begin{abstract}
The auxiliary space preconditioning (ASP) technique is applied to the HDG schemes for three different elliptic problems with a parameter dependent low order term, namely, a symmetric interior penalty HDG scheme for the scalar reaction-diffusion equation, a divergence-conforming HDG scheme for a vectorial reaction-diffusion equation, and a $C^{0}$-continuous interior penalty HDG scheme for the generalized biharmonic equation with a low order term. Uniform preconditioners are obtained for each case and the general ASP theory by J. Xu 21] is used to prove the optimality with respect to the mesh size and uniformity with respect to the low order parameter.
\end{abstract}

\section{INTRODUCTION}

The hybridizable discontinuous Galerkin (HDG) methods were originally introduced about a decade ago in [8] for diffusion problems as a subclass of discontinuous Galerkin finite element methods amenable to static condensation and hence to efficient implementation. Since then, the HDG methodology has been successfully applied to a variety of problems in computation fluid dynamics and continuum mechanics [6, $9,18,19]$.

Compared with the fast development of different HDG schemes for various partial differential equations (PDEs), there are relative few work on the issue of linear system solvers for the resulting condensed HDG system. The first investigation on fast HDG solvers appeared in [7] where the authors presented a geometric multigrid algorithm for an HDG scheme for the diffusion problem. We also mention the very recent work [15] on a multilevel HDG preconditioner that is applicable to various PDEs, and refer to [15, Section 3.1] for a short review on existing work on solvers/preconditioners for HDG systems.

The auxiliary space preconditioning (ASP) method was first proposed by $\mathrm{Xu}$ in [21], which givens an optimal (Poisson-based) preconditioner for symmetric positive definite (SPD) linear systems; see the review [22. Roughly speaking, the main idea of ASP is to use well-established fast Poisson solvers as building blocks to develop user-friendly solvers for various discretized PDEs [22]. ASP has been applied to the HDG method in [14] and the closely related weak Galerkin method in [5], both for the pure diffusion problem.

In this work, we apply ASP to HDG schemes for three different elliptic problems with a parameter dependent low order term. In particular, we consider a symmetric interior penalty HDG scheme for the scalar reaction-diffusion equation, a divergence-conforming HDG scheme for a vectorial reaction-diffusion equation, and a $C^{0}$-continuous interior penalty HDG scheme for the generalized biharmonic equation with a low order term. Uniform preconditioners are obtained for each case and the general ASP theory [21] is used to prove the optimality with respect to the mesh size and uniformity with respect to the low order parameter. We do not theoretically address the issue of robustness of the preconditioner with respect to the polynomial degree, but our numerical

2020 Mathematics Subject Classification. 65N30, 65N12, 76S05, 76D07.

Key words and phrases. Auxiliary space preconditioner, HDG methods, divergence-conforming HDG, reaction diffusion equation, biharmonic equation.

We gratefully acknowledge the partial support of this work from U.S. National Science Foundation through grant DMS-2012031. 
results indicate the growth of the iteration counts of the preconditioned conjugate gradient (PCG) algorithm on the polynomial degree is quite mild. We point out that while our analysis is focused on interior penalty type HDG schemes for each problem, our results are easily applicable to other HDG formulations and other hybrid finite element methods.

For the scalar and vectorial reaction-diffusion problems, we use the linear continuous finite element space as the auxiliary space, whose associated linear systems is precondioned by hypre's BoomerAMG preconditioner [10,11]. For the generalized biharmonic problem, we borrow the idea from the preconditioner for a divergence-free DG scheme for the Stokes problem in [3], where we take a larger auxiliary space whose associated linear system is easier to precondition than the original system for the generalized biharmonic problem. Due to a technical difficulty, the robustness of the preconditioner for the generalized biharmonic problem can only be proven for the simply supported boundary condition case, which corresponds to the slip boundary condition for Stokes flow discussed in [3]. Our numerical results confirm that our proposed preconditioner does deteriorate in performance with a mesh dependent convergence behavior when applied to the clamped boundary condition case. The construction of an optimal preconditioner for the generalized biharmonic problem with a clamped boundary condition is the subject of a forthcoming paper.

The rest of the paper is organized as follows. In Section 2, we briefly review the ASP theory in [21. In Section 3, we construct ASP for the aforementioned HDG schemes and apply the ASP theory to prove their optimality. In Section 4 , we present numerical examples to support the results in Section 3. Finally, we conclude in Section 5.

\section{ThE AUXILIARY SPACE PRECONDITIONING THEORY}

In this section, we review the abstract ASP theory developed in [21. We closely follow the discussion in [21, Section 2].

The ASP technique can be interpreted as a two level non-nested multigrid preconditioner. To be specific, we consider a finite dimensional linear inner product space $\mathcal{V}$ with an inner product $(\cdot, \cdot)$, and a linear symmetric positive definite (SPD) operator $A: \mathcal{V} \rightarrow \mathcal{V}$ with respect to this inner product. The ASP technique serves to construct an optimal (SPD) preconditioner $B$ for the operator $A$ so that mesh independent convergence behavior can be obtained for the PCG algorithm with the preconditioner $B$ for solving the linear system problem

$$
A u=f \in \mathcal{V} \text {. }
$$

The main ingredient of the ASP technique is an auxiliary linear inner product space $\mathcal{V}_{0}$ together with an operator $A_{0}: \mathcal{V}_{0} \rightarrow \mathcal{V}_{0}$ that is SPD with respect to an inner product $[\cdot, \cdot]$ on $\mathcal{V}_{0}$. The auxiliary space $\mathcal{V}_{0}$ is chosen in such a way that the operator $A_{0}$ can be more easily preconditioned than $A$.

The auxiliary space preconditioner in [21] takes the following additive form:

$$
B=R+\Pi B_{0} \Pi^{t},
$$

where $B_{0}: \mathcal{V}_{0} \rightarrow \mathcal{V}_{0}$ is an optimal preconditioner for $A_{0}, \Pi: \mathcal{V}_{0} \rightarrow \mathcal{V}$ is an operator that links the two spaces and $\Pi^{t}$ is the adjoint operator defined by

$$
\left[\Pi^{t} v, w\right]=(v, \Pi w) \quad v \in \mathcal{V}, w \in \mathcal{V}_{0},
$$

and $R: \mathcal{V} \rightarrow \mathcal{V}$ is an SPD operator serves to resolve what can not be resolved by the auxiliary space. If $\mathcal{V}_{0}$ is viewed as a coarse space in multigrid terminology, $\Pi$ can be considered as the prolongation operator, $\Pi^{t}$ is the restriction operator, $R$ is the smoother, and $B_{0}$ is the coarse grid solver. In most applications, the smoother $R$ is given by a simple relaxation scheme such as Jacobi or symmetric Gauss-Seidel method.

We cite verbatimly below the main abstract result on ASP theory [21, Theorem 2.1], which gives a sufficient condition for the optimality of the preconditioner (1). 
Theorem 2.1 (Theorem 2.1 of [21]). Assume that there are some non-negative constants $\alpha_{0}, \alpha_{1}, \lambda_{0}, \lambda_{1}$ and $\beta_{1}$ such that, for all $v \in \mathcal{V}$ and $w \in \mathcal{V}_{0}$,

$$
\begin{aligned}
\alpha_{0} \rho_{A}^{-1}(v, v) & \leq(R v, v) \leq \alpha_{1} \rho_{A}^{-1}(v, v), \\
\lambda_{0}[w, w]_{A_{0}} & \leq\left[B_{0} A_{0} w, w\right]_{A_{0}} \leq \lambda_{1}[w, w]_{A_{0}}, \\
\|\Pi w\|_{A}^{2} & \leq \beta_{1}\|w\|_{A_{0}}^{2},
\end{aligned}
$$

and furthermore, assume that there exists a linear operator $P: \mathcal{V} \rightarrow \mathcal{V}_{0}$ and positive constants $\beta_{0}$ and $\gamma_{0}$ such that,

$$
\|P v\|_{A_{0}}^{2} \leq \beta_{0}^{-1}\|v\|_{A}^{2}
$$

and

$$
\|v-\Pi P v\|^{2} \leq \gamma_{0}^{-1} \rho_{A}^{-1}\|v\|_{A}^{2} .
$$

Then the preconditioner given by (11) satisfies

$$
\kappa(B A) \leq\left(\alpha_{1}+\beta_{1} \lambda_{1}\right)\left(\left(\alpha_{0} \gamma_{0}\right)^{-1}+\left(\beta_{0} \lambda_{0}\right)^{-1}\right) .
$$

In particular, if $P$ is a right inverse of $\Pi$ namely $\Pi P v=v$ for $v \in \mathcal{V}$, then

$$
\kappa\left(\left(\Pi B_{0} \Pi^{t}\right) A\right) \leq \frac{\beta_{1}}{\beta_{0}} \frac{\lambda_{1}}{\lambda_{0}} .
$$

Here $\rho_{A}=\rho(A)$ denotes the spectral radius of $A,\|\cdot\|$ denotes the norm induced by $(\cdot, \cdot)$, the notation $(\cdot, \cdot)_{A}:=(A \cdot, \cdot)$ and $[\cdot, \cdot]_{A_{0}}:=\left[A_{0} \cdot, \cdot\right]$, and $\kappa(A)$ is the condition number of the operator $A$.

The focus of the next section is on the application of Theorems 2.1 to construct optimal auxiliary space preconditioners for the three HDG schemes under consideration. Before proceeding to the details, we make a remark below on steps in the ASP construction for the scalar and vectorial reaction-diffusion case. The ASP construction for the biharmonic case follows a different route, whose discussion is postponed to Subsection 3.4.

Remark 2.1 (ASP for HDG schemes for reaction-diffusion systems). An HDG scheme consists of two sets of degrees of freedom (DOFs): the local DOFs inside the mesh elements that can be statically condensed out, and the global DOFs on the mesh skeleton which shall form a global linear system to be solved. We denote the finite element space related to the global DOFs as $V_{h}$, and the associated HDG bilinear form on $V_{h}$ as $a_{h}(\cdot, \cdot)$. The construction of ASP for the HDG schemes for scalar and vectorial reaction-diffusion equations is performed as follows:

(i) Define an $L^{2}$-like inner-product $(\cdot, \cdot)_{0, h}$ for the global $H D G$ space $V_{h}$, and estimate the spectral radius $\rho_{A_{h}}$ of the condensed $H D G$ operator $A_{h}: V_{h} \rightarrow V_{h}$ where

$$
\left(A_{h} u, v\right)_{0, h}=a_{h}(u, v) \quad \forall u, v \in V_{h} .
$$

(ii) Prove the simple smoother $R_{h}$ given by the point Jacobi method satisfies (2a). In practical implementation, we also use the slightly more sophisticated block Gauss-Seidel method for the smoother to reduce PCG iterations for convergence.

(iii) Use the continuous piecewise linear finite element space $V_{h, 0}$ as the auxiliary space, and denote the associated bilinear form for the reaction-diffusion operator as $a_{h, 0}(\cdot, \cdot)$, with the corresponding linear operator $A_{h, 0}$ given as follows

$$
\left[A_{h, 0} u_{0}, v_{0}\right]=a_{h, 0}\left(u_{0}, v_{0}\right) \quad \forall u_{0}, v_{0} \in V_{h, 0},
$$

where $[\cdot, \cdot]$ is the usual $L^{2}$-norm on $V_{h, 0}$. Construct a robust preconditioner $B_{h, 0}$ for the operator $A_{h, 0}$ such that the estimate (2b) holds.

(iv) Construct the operators $\Pi_{h}: V_{h, 0} \rightarrow V_{h}$ and $P_{h}: V_{h} \rightarrow V_{h, 0}$ such that the stability properties (2c) $-(2 \mathrm{~d})$ and the approximation property (2e) holds.

(v) Apply Theorem 2.1 to conclude the optimality of the preconditoiner $B_{h}=R_{h}+\Pi_{h} B_{h, 0} \Pi_{h}^{t}$ for the operator $A_{h}$. 


\section{ASP FOR HDG SCHEMES}

3.1. Preliminaries and finite element spaces. We consider a convex polygonal/polyhedral domain $\Omega \subset \mathbb{R}^{d}, d=2,3$. The restrictions on the domain are only used for theoretical analysis. In practical implementation, we can work with more general non-convex or curved domains.

Let $\mathcal{T}_{h}$ be a shape-regular, quasi-uniform conforming simplicial triangulation of the domain $\Omega$. For any element $K \in \mathcal{T}_{h}$, we denote by $h_{K}$ its diameter, and by $h$ the maximum diameter over all mesh elements. We denote $\mathcal{E}_{h}$ as the set of facets (edges in 2D, faces in 3D) on the mesh $\mathcal{T}_{h}$, which we also refer to as the mesh skeleton. We split $\varepsilon_{h}$ into the collection of boundary facets $\mathcal{E}_{h}^{\partial}=\left\{F \in \mathcal{E}_{h}: F \subset \partial \Omega\right\}$, and that of the interior facets $\mathcal{E}_{h}^{o}=\mathcal{E}_{h} \backslash \mathcal{E}_{h}^{\partial}$. Given a simplex $S \subset \mathbb{R}^{d}, d=1,2,3$, we denote $\mathcal{P}^{m}(S), m \geq 0$, as the space of polynomials of degree at most $m$. Given a facet $F \in \mathcal{E}_{h}$ with normal direction $\boldsymbol{n}$, we denote $\operatorname{tang}(\boldsymbol{w}):=\boldsymbol{w}-(\boldsymbol{w} \cdot \boldsymbol{n}) \boldsymbol{n}$ as the tangential component of a vector field $\boldsymbol{w}$.

The following finite element spaces will be used to construct the HDG scheme and ASP for the scalar reaction-diffusion equation:

$$
\begin{aligned}
& W_{h}^{r}:=\left\{w \in L^{2}(\Omega):\left.\quad w\right|_{K} \in \mathcal{P}^{r}(K), \forall K \in \mathcal{T}_{h}\right\}, \\
& \widehat{W}_{h}^{r}:=\left\{\widehat{w} \in L^{2}\left(\mathcal{E}_{h}\right):\left.\widehat{w}\right|_{F} \in \mathcal{P}^{r}(F), \forall F \in \mathcal{E}_{h}\right\}, \\
& \mathcal{W}_{h}^{1}:=\left\{w \in H^{1}(\Omega):\left.\quad w\right|_{K} \in \mathcal{P}^{1}(K), \forall K \in \mathcal{T}_{h}\right\} .
\end{aligned}
$$

where $r \geq 0$ is the polynomial degree. We further denote the subspaces with vanishing boundary conditions:

$$
\widehat{W}_{h, 0}^{r}:=\left\{\widehat{w} \in \widehat{W}_{h}^{r}:\left.\widehat{w}\right|_{F}=0, \forall F \in \mathcal{E}_{h}^{\partial}\right\}, \quad \mathcal{W}_{h, 0}^{1}:=\left\{w \in \mathcal{W}_{h}^{1}:\left.w\right|_{\partial \Omega}=0\right\} .
$$

The following finite element spaces will be used to construct the divergence-conforming HDG scheme and ASP for the vectorial reaction-diffusion equation:

$$
\begin{aligned}
& \boldsymbol{V}_{h}^{r}:=\left\{\boldsymbol{v} \in H(\operatorname{div} ; \Omega):\left.\boldsymbol{v}\right|_{K} \in\left[\mathcal{P}^{r}(K)\right]^{d}, \forall K \in \mathcal{T}_{h}\right\}, \\
& \widehat{\boldsymbol{V}}_{h}^{r}:=\left\{\widehat{\boldsymbol{v}} \in\left[L^{2}\left(\mathcal{E}_{h}\right)\right]^{d}:\left.\widehat{\boldsymbol{v}}\right|_{F} \in\left[\mathcal{P}^{r}(F)\right]^{d},\left.\widehat{\boldsymbol{v}} \cdot \boldsymbol{n}\right|_{F}=0, \forall F \in \mathcal{E}_{h}\right\}, \\
& \mathcal{V}_{h}^{1}:=\left\{\boldsymbol{v} \in\left[H^{1}(\Omega)\right]^{d}:\left.\boldsymbol{v}\right|_{K} \in\left[\mathcal{P}^{1}(K)\right]^{d}, \forall K \in \mathcal{T}_{h}\right\} .
\end{aligned}
$$

We further denote the following subspaces with vanishing boundary conditions:

$$
\begin{aligned}
\boldsymbol{V}_{h, 0}^{r} & :=\left\{\boldsymbol{v} \in \boldsymbol{V}_{h}^{r}:\left.\boldsymbol{v} \cdot \boldsymbol{n}\right|_{\partial \Omega}=0\right\}, \\
\widehat{\boldsymbol{V}}_{h, 0}^{r} & :=\left\{\widehat{\boldsymbol{v}} \in \widehat{\boldsymbol{V}}_{h}^{r}:\left.\operatorname{tang}(\widehat{\boldsymbol{v}})\right|_{F}=0, \forall F \in \mathcal{E}_{h}^{\partial}\right\}, \\
\mathcal{V}_{h, 0}^{1} & :=\left\{\boldsymbol{v} \in \mathcal{V}_{h}^{1}:\left.\quad \boldsymbol{v}\right|_{\partial \Omega}=0\right\} .
\end{aligned}
$$

Moreover, we shall also use a constraint subspace of $\boldsymbol{V}_{h}^{r}$ whose elements has a piecewise constant divergence field:

$$
\begin{aligned}
\boldsymbol{V}_{h}^{r, \mathrm{cst}} & :=\left\{\boldsymbol{v} \in \boldsymbol{V}_{h}^{r}:\left.\nabla \cdot \boldsymbol{v}\right|_{K} \in \mathcal{P}^{0}(K), \forall K \in \mathcal{T}_{h}\right\}, \\
\boldsymbol{V}_{h, 0}^{r, \mathrm{cst}} & :=\left\{\boldsymbol{v} \in \boldsymbol{V}_{h, 0}^{r, \mathrm{cst}}:\left.\boldsymbol{v} \cdot \boldsymbol{n}\right|_{\partial \Omega}=0\right\} .
\end{aligned}
$$

We perform a hierarchical basis splitting for the divergence-conforming space $\boldsymbol{V}_{h}^{r}$ (and $\boldsymbol{V}_{h}^{r, \text { cst }}$ ) as was done in [23, Chapter 5], see also [13, Section 2.2.4], to facilitate the discussion on static condensation. The following basis splitting was presented in [13, Table 2.1]1]:

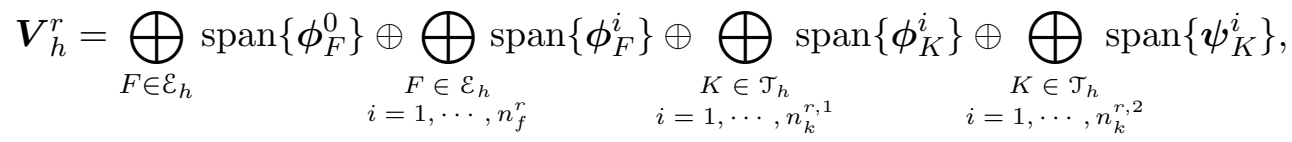

\footnotetext{
${ }^{1}$ The basis splitting in 13 is only performed on triangular meshes, similar results on other element shapes were documented in [23]. These hierarchical bases are readily available in the NGSolve software [17,20], which we use in our numerical simulations.
} 
where $\phi_{F}^{0}$ is the (global) lowest-order basis for the lowest-order Raviart-Thomas space whose normal component is only supported on the facet $F, \phi_{F}^{i}, i=1, \cdots, n_{f}^{r}$, is the (global) higher order divergence-free facet bubble basis whose normal component is only supported on the facet $F, \phi_{K}^{i}$, $i=1, \cdots, n_{k}^{r, 1}$, is the (local) higher order divergence-free element bubble basis that is supported only on the element $K$ whose normal component vanishes on the mesh skeleton $\mathcal{E}_{h}$ and $\boldsymbol{\psi}_{K}^{i}, i=$ $1, \cdots, n_{k}^{r, 2}$, is the (local) higher order element bubble basis with a non-zero divergence that is supported only on the element $K$ whose normal component vanishes on the mesh skeleton $\mathcal{E}_{h}$. Here the integer numbers $n_{f}^{r}, n_{k}^{r, 1}$, and $n_{k}^{r, 2}$ are the number of basis functions per facet/element for each group of basis functions, whose specific values can be found in [23] which are not relevant in our discussion. With this basis splitting, we now define the global and local subspaces of $\boldsymbol{V}_{h}^{r}$ :

$$
\begin{aligned}
\boldsymbol{V}_{h}^{r, \partial} & :=\bigoplus_{F \in \mathcal{E}_{h}} \operatorname{span}\left\{\boldsymbol{\phi}_{F}^{0}\right\} \oplus \underset{\substack{F \in \mathcal{E}_{h} \\
i=1, \cdots, n_{f}^{r}}}{\bigoplus} \operatorname{span}\left\{\boldsymbol{\phi}_{F}^{i}\right\} \\
\boldsymbol{V}_{h}^{r, o} & :=\bigoplus_{\substack{K \in \mathcal{T}_{h} \\
i=1, \cdots, n_{k}^{r, 1}}} \operatorname{span}\left\{\boldsymbol{\phi}_{K}^{i}\right\}, \quad \boldsymbol{V}_{h}^{r, o *}:=\boldsymbol{V}_{h}^{r, o} \oplus \bigoplus_{\substack{K \in \mathcal{T}_{h} \\
i=1, \cdots, n_{k}^{r, 2}}} \operatorname{span}\left\{\boldsymbol{\psi}_{K}^{i}\right\} .
\end{aligned}
$$

We further denote $\boldsymbol{V}_{h, 0}^{r, \partial}$ as the subspace of $\boldsymbol{V}_{h}^{r, \partial}$ whose normal component vanishes on the domain boundary $\partial \Omega$. Finally, we have the following direct decomposition of the divergence-conforming spaces that will be used in static condensation:

$$
\boldsymbol{V}_{h}^{r}=\boldsymbol{V}_{h}^{r, \partial} \oplus \boldsymbol{V}_{h}^{r, o *}, \quad \boldsymbol{V}_{h}^{r, \mathrm{cst}}=\boldsymbol{V}_{h}^{r, \partial} \oplus \boldsymbol{V}_{h}^{r, o} .
$$

The following additional high-order $H^{1}$-conforming finite element space (in two dimensions) will be used to construct the $C^{0}$-continuous interior penalty HDG scheme for the generalized biharmonic equation:

$$
X_{h}^{r}:=\left\{\phi \in H^{1}(\Omega):\left.\quad \phi\right|_{K} \in \mathcal{P}^{r}(K), \forall K \in \mathcal{T}_{h}\right\} .
$$

We further denote $X_{h, 0}^{r}$ as the subspace of $X_{h}^{r}$ with vanishing boundary conditions on the domain boundary $\partial \Omega$. Denote $V_{h, 0}^{r \text {,zero }}$ as the divergence-free subspace of $V_{h, 0}^{r}$, it is well-known that in two dimensions, the following equality holds:

$$
V_{h, 0}^{r, \text { zero }}=\left\{\vec{\nabla} \times \phi: \phi \in X_{h, 0}^{r+1}\right\}
$$

where $\vec{\nabla} \times \phi=\left(\partial_{y} \phi,-\partial_{x} \phi\right)$ is the curl operator in two dimensions.

To simplify notation, for any function $\phi \in L^{2}(S)$ we denote $\|\phi\|_{S}$ as the $L^{2}$-norm of $\phi$ on the domain $S$, and for two positive numbers $A, B \in \mathbb{R}^{+}$, we denote $A \lesssim B$ to indicate that there exists a generic positive constant $C$ that is only dependent on the shape regularity of the mesh $\mathcal{T}_{h}$ and the polynomial degree of the finite element space under consideration such that $A \leq C B$. In particular, the hidden constant $C$ is independent of the mesh size $h$ and parameters in the PDEs. Furthermore, we denote $A \simeq B$ to indicate that $A \lesssim B$ and $B \lesssim A$.

\subsection{Symmetric interior penalty $\mathrm{HDG}$ for reaction-diffusion.}

3.2.1. The model and the HDG scheme. We consider the following constant-coefficient reactiondiffusion equation with a homogeneous Dirichlet boundary condition:

$$
-\triangle u+\tau u=f \text { in } \Omega,\left.\quad u\right|_{\partial \Omega}=0
$$

where $\tau \geq 0$ is the non-negative reaction coefficient. Here we focus on the analysis for this constant coefficient case. Variable coefficients will be covered in the numerical experiments only. 
We apply the symmetric interior penalty HDG scheme with projected jumps [13, Remark 1.2.4] to discretize the equation ([6). Given a polynomial degree $k \geq 1$, the HDG scheme reads as follows: find $\left(u_{h}, \widehat{u}_{h}\right) \in W_{h}^{k} \times \widehat{W}_{h, 0}^{k-1}$ such that

$$
a_{h}\left(\left(u_{h}, \widehat{u}_{h}\right),\left(v_{h}, \widehat{v}_{h}\right)\right)=\int_{\Omega} f v_{h} \mathrm{dx}, \quad \forall\left(v_{h}, \widehat{v}_{h}\right) \in W_{h}^{k} \times \widehat{W}_{h, 0}^{k-1},
$$

where the bilinear form

$$
\begin{aligned}
a_{h}\left(\left(u_{h}, \widehat{u}_{h}\right),\left(v_{h}, \widehat{v}_{h}\right)\right): & =\sum_{K \in \mathcal{T}_{h}} \int_{K}\left(\nabla u_{h} \cdot \nabla v_{h}+\tau u_{h} v_{h}\right) \mathrm{dx}-\int_{\partial K} \nabla u_{h} \cdot \boldsymbol{n}\left(v_{h}-\widehat{v}_{h}\right) \mathrm{ds} \\
& -\int_{\partial K} \nabla v_{h} \cdot \boldsymbol{n}\left(u_{h}-\widehat{u}_{h}\right) \mathrm{ds}+\int_{\partial K} \frac{\alpha k^{2}}{h}\left(P_{k-1} u_{h}-\widehat{u}_{h}\right)\left(P_{k-1} v_{h}-\widehat{v}_{h}\right) \mathrm{ds},
\end{aligned}
$$

with $P_{k-1}$ denoting the $L^{2}$-projection into the space of facet-piecewise polynomials of degree $k-1$. Here the stability parameter $\alpha$ is chosen big enough to ensure the following coercivity result:

$$
\left\|\left(u_{h}, \widehat{u}_{h}\right)\right\|_{1, h}^{2} \lesssim a_{h}\left(\left(u_{h}, \widehat{u}_{h}\right),\left(u_{h}, \widehat{u}_{h}\right)\right),
$$

where

$$
\left\|\left(u_{h}, \widehat{u}_{h}\right)\right\|_{1, h}^{2}:=\sum_{K \in \mathcal{T}_{h}}\left(\left\|\nabla u_{h}\right\|_{K}^{2}+\tau\left\|u_{h}\right\|_{K}^{2}+\frac{1}{h}\left\|u_{h}-\widehat{u}_{h}\right\|_{\partial K}^{2}\right)
$$

is a norm on $W_{h}^{k} \times \widehat{W}_{h, 0}^{k-1}$. A lower bound on $\alpha$ that guarantees coercivity was presented in [1, Lemma 1]. In practice, taking $\alpha=4$ ensures (8), which is the value we use in the numerical simulations. On the other hand, the Cauchy-Schwarz inequality and inverse inequality ensure that $a_{h}\left(\left(u_{h}, \widehat{u}_{h}\right),\left(u_{h}, \widehat{u}_{h}\right)\right) \lesssim \|\left(u_{h}, \widehat{u}_{h} \|_{1, h}^{2}\right.$ for $\left(u_{h}, \widehat{u}_{h}\right) \in W_{h}^{k} \times W_{h, 0}^{k-1}$. Hence, the bilinear form $a_{h}$ induces a norm that is equivalent to $\|\cdot\|_{1, h}$ :

$$
a_{h}\left(\left(u_{h}, \widehat{u}_{h}\right),\left(u_{h}, \widehat{u}_{h}\right)\right) \simeq\left\|\left(u_{h}, \widehat{u}_{h}\right)\right\|_{1, h}^{2}, \quad \forall\left(u_{h}, \widehat{u}_{h}\right) \in W_{h}^{k} \times \widehat{W}_{h, 0}^{k-1} .
$$

3.2.2. Static condensation. When implementing the scheme (7), static condensation is performed locally in the element-level to eliminate DOFs associated with the local space $W_{h}^{k}$, which results in a global condense linear system for DOFs associated with the global space $\widehat{W}_{h, 0}^{k-1}$ only. The main subject of this subsection is to construct an ASP method for this condensed system. To proceed, we first give a characterization of the condensed bilinear form.

Denoting the following lifting operator $\mathcal{L}_{h}: \widehat{W}_{h, 0}^{k-1} \rightarrow W_{h}^{k}$ : given $\mu \in \widehat{W}_{h, 0}^{k-1}, \mathcal{L}_{h}(\mu)$ is the unique function in $W_{h}^{k}$ such that

$$
a_{h}\left(\left(\mathcal{L}_{h}(\mu), 0\right),\left(v_{h}, 0\right)\right)=-a_{h}\left((0, \mu),\left(v_{h}, 0\right)\right), \quad \forall v_{h} \in W_{h}^{k} .
$$

Furthermore, let $\mathcal{L}_{h}^{f}$ be the unique function in $W_{h}^{k}$ such that

$$
a_{h}\left(\left(\mathcal{L}_{h}^{f}, 0\right),\left(v_{h}, 0\right)\right)=\int_{\Omega} f v_{h} \mathrm{dx}, \forall v_{h} \in W_{h}^{k}
$$

Well-posedness of the above linear systems easily follows from the coercivity result (8) $)$.

Lemma 3.1 (Characterization of the condensed system for (17)). Let $\left(u_{h}, \widehat{u}_{h}\right) \in W_{h}^{k} \times \widehat{W}_{h, 0}^{k-1}$ be the unique solution to the HDG scheme (7). Then,

$$
u_{h}=\mathcal{L}_{h}\left(\widehat{u}_{h}\right)+\mathcal{L}_{h}^{f},
$$

and $\widehat{u}_{h}$ is the unique function in $\widehat{W}_{h, 0}^{k-1}$ such that

$$
a_{h}\left(\left(\mathcal{L}_{h}\left(\widehat{u}_{h}\right), \widehat{u}_{h}\right),\left(\mathcal{L}_{h}\left(\widehat{v}_{h}\right), \widehat{v}_{h}\right)\right)=-a_{h}\left(\left(\mathcal{L}_{h}^{f}, 0\right),\left(\mathcal{L}_{h}\left(\widehat{v}_{h}\right), \widehat{v}_{h}\right)\right), \quad \forall \widehat{v}_{h} \in \widehat{W}_{h, 0}^{k-1} .
$$


In particular, the reduced system (12b) is symmetric and positive definite, and

$$
a_{h}\left(\left(\mathcal{L}_{h}(\mu), \mu\right),\left(\mathcal{L}_{h}(\mu), \mu\right)\right) \simeq\left\|\left(\mathcal{L}_{h}(\mu), \mu\right)\right\|_{1, h}^{2}, \quad \forall \mu \in \widehat{W}_{h, 0}^{k-1} .
$$

Proof. Taking test functions $\left(v_{h}, 0\right)$ in the HDG scheme (7), we immediately obtain the equality (12a) from the definitions (10)-(11) and linearity of the bilinear form $a_{h}$. Taking test functions $\left(\mathcal{L}_{h}\left(\widehat{v}_{h}\right), \widehat{v}_{h}\right)$ in (7) and reordering terms, we obtain the equation (12b). Finally, the equivalence (12c) is a consequence of that for the operator $a_{h}$ in (9).

3.2.3. The auxiliary space preconditioner. Now, we follow Remark 2.1 to construct the auxiliary space perconditioner for the reduced HDG system (12b).

(i) We denote the following $L^{2}$-like inner product on $\widehat{W}_{h, 0}^{k-1}$ :

$$
(\lambda, \mu)_{0, h}:=\sum_{K \in \mathcal{T}_{h}} h \int_{\partial K} \lambda \mu \mathrm{ds}=\sum_{F \in \mathcal{E}_{h}^{o}} h \int_{F} \lambda \mu \mathrm{ds}, \quad \forall \lambda, \mu \in \widehat{W}_{h, 0}^{k-1},
$$

whose induced norm is denoted as $\|\mu\|_{0, h}^{2}:=(\mu, \mu)_{0, h}$. We define the condensed HDG operator $A_{h}: \widehat{W}_{h, 0}^{k-1} \rightarrow \widehat{W}_{h, 0}^{k-1}$ as

$$
\left(A_{h} \widehat{u}_{h}, \widehat{v}_{h}\right)_{0, h}:=a_{h}\left(\left(\mathcal{L}_{h}\left(\widehat{u}_{h}\right), \widehat{u}_{h}\right),\left(\mathcal{L}_{h}\left(\widehat{v}_{h}\right), \widehat{v}_{h}\right)\right), \quad \forall \widehat{u}_{h}, \widehat{v}_{h} \in \widehat{W}_{h, 0}^{k-1} .
$$

The following result gives an estimation of the spectral radius of $A_{h}$ and its condition number.

Lemma 3.2 (Spectral radius and condition number). Let $A_{h}$ be the operator given in (14). Then, there holds $\rho_{A_{h}} \simeq h^{-2}$ and $\kappa\left(A_{h}\right) \lesssim \frac{h^{-2}}{\min \left\{\tau+1, h^{-2}\right\}}$.

Proof. First, we prove $\rho_{A_{h}} \lesssim h^{-2}$. By the definition of the operator $A_{h}$, we have $\left(A_{h} \widehat{u}_{h}, \widehat{u}_{h}\right)_{0, h} \simeq$ $\left\|\left(\mathcal{L}_{h}\left(\widehat{u}_{h}\right), \widehat{u}_{h}\right)\right\|_{1, h}^{2}$. Taking $\mu=\widehat{u}_{h}$ and $v_{h}=\mathcal{L}_{h}\left(\widehat{u}_{h}\right)$ in (10) and applying the Cauchy-Schwarz inequality, we have

$$
\begin{aligned}
a_{h}\left(\left(\mathcal{L}_{h}\left(\widehat{u}_{h}\right), 0\right),\left(\mathcal{L}_{h}\left(\widehat{u}_{h}\right), 0\right)\right)=-a_{h}\left(\left(0, \widehat{u}_{h}\right),\left(\mathcal{L}_{h}\left(\widehat{u}_{h}\right), 0\right)\right) \\
\leq a_{h}\left(\left(\mathcal{L}_{h}\left(\widehat{u}_{h}\right), 0\right),\left(\mathcal{L}_{h}\left(\widehat{u}_{h}\right), 0\right)\right)^{1 / 2} a_{h}\left(\left(0, \widehat{u}_{h}\right),\left(0, \widehat{u}_{h}\right)\right)^{1 / 2} .
\end{aligned}
$$

Hence,

$$
\left\|\left(\mathcal{L}_{h}\left(\widehat{u}_{h}\right), 0\right)\right\|_{1, h}^{2} \simeq a_{h}\left(\left(\mathcal{L}_{h}\left(\widehat{u}_{h}\right), 0\right),\left(\mathcal{L}_{h}\left(\widehat{u}_{h}\right), 0\right)\right) \lesssim a_{h}\left(\left(0, \widehat{u}_{h}\right),\left(0, \widehat{u}_{h}\right)\right) \simeq\left\|\left(0, \widehat{u}_{h}\right)\right\|_{1, h}^{2} .
$$

Invoking the triangle inequality, we get

$$
\left(A_{h} \widehat{u}_{h}, \widehat{u}_{h}\right)_{0, h} \simeq\left\|\left(\mathcal{L}_{h}\left(\widehat{u}_{h}\right), \widehat{u}_{h}\right)\right\|_{1, h}^{2} \lesssim\left\|\left(0, \widehat{u}_{h}\right)\right\|_{1, h}^{2}=h^{-2}\left\|\widehat{u}_{h}\right\|_{0, h}^{2},
$$

where the last equality following from the definition of these norms. Since the spectral radius of the SPD operator $A_{h}$ is its maximum eigenvalue, $\lambda_{A_{h}}^{\max }$, we conclude that $\rho_{A_{h}}=\lambda_{A_{h}}^{\max } \lesssim h^{-2}$.

Next, we prove the condition number estimate. By inverse and triangle inequalities, we get, for any $\widehat{u}_{h} \in \widehat{W}_{h, 0}^{k-1}$,

$$
\begin{aligned}
\min \left\{\tau+1, h^{-2}\right\}\left\|\widehat{u}_{h}\right\|_{0, h}^{2} & \lesssim(\tau+1)\left\|\mathcal{L}_{h}\left(\widehat{u}_{h}\right)\right\|_{0, h}^{2}+h^{-2}\left\|\mathcal{L}_{h}\left(\widehat{u}_{h}\right)-\widehat{u}_{h}\right\|_{0, h}^{2} \\
& \lesssim(\tau+1)\left\|\mathcal{L}_{h}\left(\widehat{u}_{h}\right)\right\|_{\Omega}^{2}+h^{-2}\left\|\mathcal{L}_{h}\left(\widehat{u}_{h}\right)-\widehat{u}_{h}\right\|_{0, h}^{2} \\
& \lesssim\left\|\left(\mathcal{L}_{h}\left(\widehat{u}_{h}\right), \widehat{u}_{h}\right)\right\|_{1, h}^{2} \simeq\left(A_{h} \widehat{u}_{h}, \widehat{u}_{h}\right)_{0, h},
\end{aligned}
$$

where in the last step, we invoked the following discrete Poincaré inequality

$$
\left\|\mathcal{L}_{h}\left(\widehat{u}_{h}\right)\right\|_{\Omega} \lesssim\left\|\left(\mathcal{L}_{h}\left(\widehat{u}_{h}\right), \widehat{u}_{h}\right)\right\|_{1, h},
$$


which holds on convex domains, see [2, Lemma 2.1]. Hence, the minimum eigenvalue $\lambda_{A_{h}}^{\min }$ of $A_{h}$ satisfies $\min \left\{\tau+1, h^{-2}\right\} \lesssim \lambda_{A_{h}}^{\min }$. This implies that

$$
\kappa\left(A_{h}\right)=\frac{\lambda_{A_{h}}^{\max }}{\lambda_{A_{h}}^{\min }} \lesssim \frac{h^{-2}}{\min \left\{\tau+1, h^{-2}\right\}} .
$$

Finally, we prove $h^{-2} \lesssim \rho_{A_{h}}$. Take $\mu$ as a basis function of $\widehat{W}_{h, 0}^{k-1}$ that is supported on a single facet $F$, then norm equivalence of finite dimensional spaces and the standard scaling argument implies that

$$
\left(A_{h} \mu, \mu\right)_{0, h} \simeq h^{-2}\|\mu\|_{0, h}^{2}
$$

This concludes the proof.

Remark 3.1 (The case with a large reaction parameter). When the reaction parameter $\tau \gtrsim h^{-2}$, Lemma 3.2 implies that $\kappa\left(A_{h}\right) \simeq 1$. In this case, a simple Jacobi preconditioner is already an optimal one for $A_{h}$.

(ii) With the above spectral radius estimate, we now show next that the simple Jacobi smoother satisfies (2a).

Lemma 3.3 (Spectum of the Jacobi preconditioner). Let $\left\{\phi_{F}^{j}: F \in \mathcal{E}_{h}^{o}, 1 \leq j \leq N_{f}\right\}$ be a set of orthogonal bases for $\widehat{W}_{h, 0}^{k-1}$ with respect to the inner product $(\cdot, \cdot)_{0, h}$. Here $\phi_{F}^{j}$ is supported only on the facet $F$, and $N_{f}=\left(\begin{array}{c}k+d-1 \\ d-1\end{array}\right)$ is the number of bases per facet. Denote $R_{h}: \widehat{W}_{h, 0}^{k-1} \rightarrow \widehat{W}_{h, 0}^{k-1}$ as the Jacobi preconditioner for $A_{h}$ associated with this basis set, i.e., the operator $R_{h}^{-1}$ is the diagonal component of $A_{h}$ :

$$
\left(R_{h}^{-1} \widehat{u}_{h}, \widehat{v}_{h}\right)_{0, h}=\sum_{F \in \mathcal{E}_{h}^{o}} \sum_{j=1}^{N_{f}} \mathrm{u}_{F}^{j} \mathrm{v}_{F}^{j}\left(A_{h} \phi_{F}^{j}, \phi_{F}^{j}\right)_{0, h},
$$

where $\widehat{u}_{h}=\sum_{F \in \mathcal{E}_{h}^{o}} \sum_{j=1}^{N_{f}} \mathrm{u}_{F}^{j} \phi_{F}^{j}$ and $\widehat{v}_{h}=\sum_{F \in \mathcal{E}_{h}^{o}} \sum_{j=1}^{N_{f}} \mathrm{v}_{F}^{j} \phi_{F}^{j}$ with $\mathrm{u}_{F}^{j} \in \mathbb{R}$ and $\mathrm{v}_{F}^{j} \in \mathbb{R}$ being the basis coefficients of $\widehat{u}_{h}$ and $\widehat{v}_{h}$, respectively. Then, the following equivalence holds:

$$
\left(R_{h} \widehat{u}_{h}, \widehat{u}_{h}\right)_{0, h} \simeq \rho_{A_{h}}^{-1}\left(\widehat{u}_{h}, \widehat{u}_{h}\right)_{0, h} .
$$

Proof. It suffice to show

$$
\left(R_{h}^{-1} \widehat{u}_{h}, \widehat{u}_{h}\right)_{0, h} \simeq \rho_{A_{h}}\left(\widehat{u}_{h}, \widehat{u}_{h}\right)_{0, h} .
$$

By the definition (16) and the assumption of the bases, we have

$$
\begin{aligned}
& \left(R_{h}^{-1} \widehat{u}_{h}, \widehat{u}_{h}\right)_{0, h}=\sum_{F \in \mathcal{E}_{h}^{o}} \sum_{j=1}^{N_{f}}\left(\mathrm{u}_{F}^{j}\right)^{2}\left(A_{h} \phi_{F}^{j}, \phi_{F}^{j}\right)_{0, h} \\
& \simeq h^{-2} \sum_{F \in \mathcal{E}_{h}^{o}} \sum_{j=1}^{N_{f}}\left(\mathbf{u}_{F}^{j}\right)^{2}\left\|\phi_{F}^{j}\right\|_{0, h}^{2} \quad \text { by (35) } \\
& =h^{-2}\left\|\widehat{u}_{h}\right\|_{0, h}^{2} \simeq \rho_{A_{h}}\left\|\widehat{u}_{h}\right\|_{0, h}^{2} \quad \text { by basis orthogonality. }
\end{aligned}
$$

This completes the proof. 
Remark 3.2 (On the smoother). The above result also holds if we use other basis functions for $\widehat{W}_{h, 0}^{k-1}$ like the nodal Lagrange basis, where we would have

$$
\sum_{F \in \mathcal{E}_{h}^{o}} \sum_{j=1}^{N_{f}}\left(\mathrm{u}_{F}^{j}\right)^{2}\left\|\phi_{F}^{j}\right\|_{0, h}^{2} \simeq\left\|\widehat{u}_{h}\right\|_{0, h}^{2}
$$

following from the norm equivalence of a finite dimensional space and a scaling argument.

This result also implies that the symmetric Gauss-Seidel smoother satisfies (2a) as it is spectrally equivalent to the Jacobi smoother [24]. In practice, we prefer to use a block version of these smoothers as they usually lead to better convergence for the PCG algorithm.

(iii) The auxiliary space under consideration is the continuous piecewise linear finite element space $\mathcal{W}_{h, 0}^{1}$ in (3d). The associated bilinear form $a_{h, 0}$ and the linear operator $A_{h, 0}$ are given as follows:

$$
\left[A_{h, 0} u_{0}, v_{0}\right]=a_{h, 0}\left(u_{0}, v_{0}\right):=\int_{\Omega}\left(\nabla u_{0} \cdot \nabla v_{0}+\tau u_{0} v_{0}\right) \mathrm{dx}, \quad \forall u_{0}, v_{0} \in \mathcal{W}_{h, 0}^{1},
$$

where $[\cdot, \cdot]$ is the usual $L^{2}$-norm on $\mathcal{W}_{h, 0}^{1}$. The construction of a robust preconditioner $B_{h, 0}$ for the operator $A_{h, 0}$ is a well-studied subject in the literature. Here we take $B_{h, 0}$ to be hypre's BoomerAMG preconditioner [10,11], which is numerically verified to be robust with respect to both mesh size $h$ and reaction parameter $\tau$.

(iv) We take the operator $\Pi_{h}: \mathcal{W}_{h, 0}^{1} \rightarrow \widehat{W}_{h, 0}^{k-1}$ to be the simple $L^{2}$-projection:

$$
\left(\Pi_{h} u_{0}, \widehat{v}_{h}\right)_{0, h}=\left(u_{0}, \widehat{v}_{h}\right)_{0, h}, \quad \forall \widehat{v}_{h} \in \widehat{W}_{h, 0}^{k-1},
$$

and the operator $P_{h}: \widehat{W}_{h, 0}^{k-1} \rightarrow \mathcal{W}_{h, 0}^{1}$ to be

$$
P_{h}\left(\widehat{u}_{h}\right)=S_{1}\left(\mathcal{L}_{h}\left(\widehat{u}_{h}\right)\right),
$$

where $S_{1}: W_{h}^{k} \rightarrow \mathcal{W}_{h, 0}^{1}$ is the simple nodal average: given any $v \in W_{h}^{k}, S_{1}(v) \in \mathcal{W}_{h, 0}^{1}$ is defined through its vertex values as follows,

$$
S_{1}(v)\left(\boldsymbol{x}_{n}\right)= \begin{cases}0 & \text { if } \boldsymbol{x}_{n} \in \partial \Omega, \\ \left.\frac{1}{\# K_{n}} \sum_{K \subset K_{n}} v\right|_{K}\left(\boldsymbol{x}_{n}\right) & \text { if } \boldsymbol{x}_{n} \notin \partial \Omega,\end{cases}
$$

where $\boldsymbol{x}_{n}$ is a vertex of the mesh $\mathcal{T}_{h}, K_{n}$ is the set of elements that have a vertex at the point $\boldsymbol{x}_{n}$, and $\# K_{n}$ denotes its cardinality.

The following result shows that $\Pi_{h}$ and $P_{h}$ satisfies both the stability and approximation properties needed to apply the ASP theory, Theorem 2.1.

Lemma 3.4 (Properties of $\Pi_{h}$ and $P_{h}$ ). Let the operator $\Pi_{h}$ be given in (18) and $P_{h}$ be given in (19). Then the following inequalities holds

$$
\begin{aligned}
\left\|\Pi_{h} u_{0}\right\|_{A_{h}} & \lesssim\left\|u_{0}\right\|_{A_{h, 0}}, \\
\left\|P_{h} \widehat{u}_{h}\right\|_{A_{h, 0}} & \lesssim\left\|\widehat{u}_{h}\right\|_{A_{h}}, \\
\left\|\widehat{u}_{h}-\Pi_{h} P_{h} \widehat{u}_{h}\right\|_{0, h} & \lesssim \rho_{A_{h}}^{-1 / 2}\left\|\widehat{u}_{h}\right\|_{A_{h}},
\end{aligned}
$$

where

$$
\left\|\widehat{v}_{h}\right\|_{A_{h}}^{2}:=a_{h}\left(\left(\mathcal{L}_{h}\left(\widehat{v}_{h}\right), \widehat{v}_{h}\right),\left(\mathcal{L}_{h}\left(\widehat{v}_{h}\right), \widehat{v}_{h}\right)\right) \text {, and }\left\|v_{0}\right\|_{A_{h, 0}}^{2}:=a_{h, 0}\left(v_{0}, v_{0}\right) .
$$

Proof. By the definition of $\Pi_{h}$ in (18), there holds

$$
\left\|\Pi_{h} u_{0}-u_{0}\right\|_{0, h}=\inf _{v_{0} \in \widehat{W}_{h, 0}^{k-1}}\left\|u_{0}-v_{0}\right\|_{0, h} \leq\left\|u_{0}-\bar{u}_{0}\right\|_{0, h} \lesssim\left\|u_{0}-\bar{u}_{0}\right\|_{\Omega} \lesssim h\left\|\nabla u_{0}\right\|_{\Omega},
$$

where $\bar{u}_{0} \in W_{h}^{0}$ is the $L^{2}$-projection of $u_{0}$ into the piecewise constant space $W_{h}^{0}$. 
Next, we prove

$$
\left\|u_{0}\right\|_{A_{h}} \lesssim\left\|u_{0}\right\|_{A_{h, 0},}, \quad \forall u_{0} \in \mathcal{W}_{h, 0}^{1},
$$

where $u_{0}$ on the left hand side is understood as a function on the mesh skeleton. By (12c), we have

$$
\left\|u_{0}\right\|_{A_{h}} \simeq\left\|\left(\mathcal{L}_{h}\left(u_{0}\right), u_{0}\right)\right\|_{1, h}
$$

On the other hand, definition of the lifting operator (10) implies, for any $v_{h} \in W_{h}^{k}$,

$$
a_{h}\left(\left(\mathcal{L}_{h}\left(u_{0}\right)-u_{0}, 0\right),\left(v_{h}, 0\right)\right)=-a_{h}\left(\left(u_{0}, u_{0}\right),\left(v_{h}, 0\right)\right)
$$

Taking $v_{h}=\mathcal{L}_{h}\left(u_{0}\right)-u_{0}$ in the above equation and applying the Cauchy-Schwartz inequality, we get

$$
\left\|\left(\mathcal{L}_{h}\left(u_{0}\right)-u_{0}, 0\right)\right\|_{1, h}^{2} \lesssim\left\|\left(u_{0}, u_{0}\right)\right\|_{1, h}^{2}=\left\|u_{0}\right\|_{A_{h, 0}}^{2},
$$

where the last step follows from definition of the norms. The estimate (23) then follows from the triangle inequality and the above inequality.

Now the inequality (21a) follows from (22)-(23) and $\rho_{A_{h}} \simeq h^{-2}$ (from Lemma 3.2):

$$
\left\|\Pi_{h} u_{0}\right\|_{A_{h}} \leq\left\|\Pi_{h} u_{0}-u_{0}\right\|_{A_{h}}+\left\|u_{0}\right\|_{A_{h}} \lesssim h^{-1}\left\|\Pi_{h} u_{0}-u_{0}\right\|_{0, h}+\left\|u_{0}\right\|_{A_{h, 0}} \lesssim\left\|u_{0}\right\|_{A_{h, 0}} .
$$

To prove the inequality (21b), we use the following result for the averaging operator $S_{1}$

$$
\left\|P_{h} \widehat{u}_{h}-\mathcal{L}_{h}\left(\widehat{u}_{h}\right)\right\|_{\Omega}^{2} \lesssim h^{2}\left\|\nabla \mathcal{L}_{h}\left(\widehat{u}_{h}\right)\right\|_{\mathcal{T}_{h}}^{2}+\left\|\llbracket \mathcal{L}_{h}\left(\widehat{u}_{h}\right) \rrbracket\right\|_{0, h}^{2}
$$

where $\llbracket \cdot \rrbracket$ is the jump on interior facets. Then the inverse inequality implies that

$$
\left\|P_{h} \widehat{u}_{h}-\mathcal{L}_{h}\left(\widehat{u}_{h}\right)\right\|_{\Omega} \lesssim\left\|\mathcal{L}_{h}\left(\widehat{u}_{h}\right)\right\|_{\Omega},
$$

and definition of the norm $\|\cdot\|_{A_{h}}$ implies that

$$
\left\|P_{h} \widehat{u}_{h}-\mathcal{L}_{h}\left(\widehat{u}_{h}\right)\right\|_{\Omega} \lesssim h\left\|\widehat{u}_{h}\right\|_{A_{h}} .
$$

Hence, we have

$$
\begin{array}{rlrl}
\left\|P_{h} \widehat{u}_{h}\right\|_{A_{h, 0}}^{2} & \lesssim\left\|P_{h} \widehat{u}_{h}-\mathcal{L}_{h}\left(\widehat{u}_{h}\right)\right\|_{A_{h, 0}}^{2}+\left\|\mathcal{L}_{h}\left(\widehat{u}_{h}\right)\right\|_{A_{h, 0}}^{2} \\
& =\left\|\nabla\left(P_{h} \widehat{u}_{h}-\mathcal{L}_{h}\left(\widehat{u}_{h}\right)\right)\right\|_{\mathcal{T}_{h}}^{2}+\tau\left\|P_{h} \widehat{u}_{h}-\mathcal{L}_{h}\left(\widehat{u}_{h}\right)\right\|_{\Omega}^{2}+\left\|\mathcal{L}_{h}\left(\widehat{u}_{h}\right)\right\|_{A_{h, 0}}^{2} \\
& \lesssim h^{-2}\left\|P_{h} \widehat{u}_{h}-\mathcal{L}_{h}\left(\widehat{u}_{h}\right)\right\|_{\Omega}^{2}+\left\|\mathcal{L}_{h}\left(\widehat{u}_{h}\right)\right\|_{A_{h, 0}}^{2} & \text { by the inverse inequality and (24) }, \\
& \lesssim\left\|\widehat{u}_{h}\right\|_{A_{h}}^{2} & \text { by the definition of norms and (25). }
\end{array}
$$

Finally, let us prove the approximation property (21c).

$$
\begin{aligned}
\left\|\widehat{u}_{h}-\Pi_{h} P_{h} \widehat{u}_{h}\right\|_{0, h} & \lesssim\left\|\widehat{u}_{h}-\mathcal{L}_{h}\left(\widehat{u}_{h}\right)\right\|_{0, h}+\left\|\mathcal{L}_{h}\left(\widehat{u}_{h}\right)-P_{h} \widehat{u}_{h}\right\|_{0, h}+\left\|P_{h} \widehat{u}_{h}-\Pi_{h} P_{h} \widehat{u}_{h}\right\|_{0, h} \\
& \lesssim h\left\|\widehat{u}_{h}\right\|_{A_{h}}+\left\|\mathcal{L}_{h}\left(\widehat{u}_{h}\right)-P_{h} \widehat{u}_{h}\right\|_{\Omega}+h\left\|\nabla\left(P_{h} \widehat{u}_{h}\right)\right\|_{\Omega} \\
& \lesssim h\left\|\widehat{u}_{h}\right\|_{A_{h}}+\left\|\mathcal{L}_{h}\left(\widehat{u}_{h}\right)-P_{h} \widehat{u}_{h}\right\|_{\Omega}+h\left\|\nabla\left(\mathcal{L}_{h}\left(\widehat{u}_{h}\right)\right)\right\|_{\mathcal{T}_{h}} \\
& \lesssim h\left\|\widehat{u}_{h}\right\|_{A_{h}},
\end{aligned}
$$

where the first step follows from the triangle inequality, the second step follows from the inverse inequality and (22), the third step follows from the triangle inequality and inverse inequality, and the last step follows from (25) and definition of the $A_{h}$-norm.

(v) Finally, we conclude the following optimality of the auxiliary space preconditioner by invoking Theorem 2.1 and applying Lemma 3.2-Lemma 3.4.

Theorem 3.1 (ASP for the condensed HDG operator (14)). Let $B_{h}=R_{h}+\Pi_{h} B_{h, 0} \Pi_{h}^{t}$ be the auxiliary space preconditioner for the operator $A_{h}$ in (14) with $R_{h}$ being the Jacobi smoother for $A_{h}, B_{h, 0}$ being an optimal preconditioner for $A_{h, 0}$ in (17) such that $\kappa\left(B_{h, 0} A_{h, 0}\right) \simeq 1$, and $\Pi_{h}$ being the projector in (18). Then, $\kappa\left(B_{h} A_{h}\right) \simeq 1$.

\subsection{Divergence-conforming HDG for vectorial reaction-diffusion.}


3.3.1. The model and the HDG scheme. We consider the following constant-coefficient vectorial reaction-diffusion equation with a homogeneous Dirichlet boundary condition:

$$
-\triangle \boldsymbol{u}+\tau \boldsymbol{u}=\boldsymbol{f} \text { in } \Omega,\left.\quad \boldsymbol{u}\right|_{\partial \Omega}=0
$$

where $\tau \geq 0$ is the non-negative reaction coefficient. Here $\boldsymbol{u}: \Omega \rightarrow \mathbb{R}^{d}$ is the solution to be approximated. Again, our analysis focuses on this constant coefficient case, while variable coefficients will be covered in the numerical experiments.

We apply the (interior penalty based) divergence-conforming HDG scheme with projected jumps [13, Chapter 2] to discretize the equation (26). Given a polynomial degree $k \geq 1$, the HDG scheme reads as follows: find $\left(\boldsymbol{u}_{h}, \widehat{\boldsymbol{u}}_{h}\right) \in \boldsymbol{V}_{h, 0}^{k} \times \widehat{\boldsymbol{V}}_{h, 0}^{k-1}$ such that

$$
\boldsymbol{a}_{h}\left(\left(\boldsymbol{u}_{h}, \widehat{\boldsymbol{u}}_{h}\right),\left(\boldsymbol{v}_{h}, \widehat{\boldsymbol{v}}_{h}\right)\right)=\int_{\Omega} \boldsymbol{f} \cdot \boldsymbol{v}_{h} \mathrm{dx}, \quad \forall\left(\boldsymbol{v}_{h}, \widehat{\boldsymbol{v}}_{h}\right) \in \boldsymbol{V}_{h, 0}^{k} \times \widehat{\boldsymbol{V}}_{h, 0}^{k-1},
$$

where the bilinear form

$$
\begin{aligned}
\boldsymbol{a}_{h}\left(\left(\boldsymbol{u}_{h}, \widehat{\boldsymbol{u}}_{h}\right),\left(\boldsymbol{v}_{h}, \widehat{\boldsymbol{v}}_{h}\right)\right):=\sum_{K \in \mathcal{T}_{h}} \int_{K}\left(\nabla \boldsymbol{u}_{h}: \nabla \boldsymbol{v}_{h}+\tau \boldsymbol{u}_{h} \cdot \boldsymbol{v}_{h}\right) \mathrm{dx}-\int_{\partial K}\left(\nabla \boldsymbol{u}_{h}\right) \boldsymbol{n} \cdot \operatorname{tang}\left(\boldsymbol{v}_{h}-\widehat{\boldsymbol{v}}_{h}\right) \mathrm{ds} \\
-\int_{\partial K}\left(\nabla \boldsymbol{v}_{h}\right) \boldsymbol{n} \cdot \operatorname{tang}\left(\boldsymbol{u}_{h}-\widehat{\boldsymbol{u}}_{h}\right) \mathrm{ds}+\int_{\partial K} \frac{\alpha k^{2}}{h} \operatorname{tang}\left(\boldsymbol{P}_{k-1} \boldsymbol{u}_{h}-\widehat{\boldsymbol{u}}_{h}\right) \cdot \operatorname{tang}\left(\boldsymbol{P}_{k-1} \boldsymbol{v}_{h}-\widehat{\boldsymbol{v}}_{h}\right) \mathrm{ds},
\end{aligned}
$$

with $\boldsymbol{P}_{k-1}$ denoting the $L^{2}$-projection into the space of vectorial facet-piecewise polynomials of degree $k-1$. Here, again, we take the stability parameter $\alpha=4$ to ensure coercivity. In particular, we have the following norm equivalence result:

$$
\boldsymbol{a}_{h}\left(\left(\boldsymbol{u}_{h}, \widehat{\boldsymbol{u}}_{h}\right),\left(\boldsymbol{u}_{h}, \widehat{\boldsymbol{u}}_{h}\right)\right) \simeq\left\|\left(\boldsymbol{u}_{h}, \widehat{\boldsymbol{u}}_{h}\right)\right\|_{1, h}^{2}, \quad \forall\left(\boldsymbol{u}_{h}, \widehat{\boldsymbol{u}}_{h}\right) \in \boldsymbol{V}_{h, 0}^{k} \times \widehat{\boldsymbol{V}}_{h, 0}^{k-1}
$$

where

$$
\left\|\left(\boldsymbol{u}_{h}, \widehat{\boldsymbol{u}}_{h}\right)\right\|_{1, h}^{2}:=\sum_{K \in \mathcal{T}_{h}}\left(\left\|\nabla \boldsymbol{u}_{h}\right\|_{K}^{2}+\tau\left\|\boldsymbol{u}_{h}\right\|_{K}^{2}+\frac{1}{h}\left\|\boldsymbol{u}_{h}-\widehat{\boldsymbol{u}}_{h}\right\|_{\partial K}^{2}\right)
$$

is a norm on $\boldsymbol{V}_{h, 0}^{k} \times \widehat{\boldsymbol{V}}_{h, 0}^{k-1}$.

3.3.2. Static condensation. Static condensation for the scheme (27) is similar to that for the scalar case (7), but the notation is a bit more involved.

We use the space splitting in (41) to perform static condensation that locally eliminate DOFs associated with the bubble space $\boldsymbol{V}_{h}^{k, o *}$. Denoting the following lifting operator $\mathcal{L}_{h}: \boldsymbol{V}_{h, 0}^{k, \partial} \times \widehat{\boldsymbol{V}}_{h, 0}^{k-1} \rightarrow$ $\boldsymbol{V}_{h}^{k, o *}$ : given $\left(\boldsymbol{w}_{h}^{\partial}, \widehat{\boldsymbol{w}}_{h}\right) \in \boldsymbol{V}_{h, 0}^{k, \partial} \times \widehat{\boldsymbol{V}}_{h, 0}^{k-1}, \mathcal{L}_{h}\left(\boldsymbol{w}_{h}^{\partial}, \widehat{\boldsymbol{w}}_{h}\right)$ is the unique function in $\boldsymbol{V}_{h}^{k, o *}$ such that

$$
\boldsymbol{a}_{h}\left(\left(\mathcal{L}_{h}\left(\boldsymbol{w}_{h}^{\partial}, \widehat{\boldsymbol{w}}_{h}\right), 0\right),\left(\boldsymbol{v}_{h}^{o}, 0\right)\right)=-\boldsymbol{a}_{h}\left(\left(\boldsymbol{w}_{h}^{\partial}, \widehat{\boldsymbol{w}}_{h}\right),\left(\boldsymbol{v}_{h}^{o}, 0\right)\right), \quad \forall \boldsymbol{v}_{h}^{o} \in \boldsymbol{V}_{h}^{k, o *},
$$

and let $\mathcal{L}_{h}^{f}$ be the unique function in $\boldsymbol{V}_{h}^{k, o *}$ such that

$$
\boldsymbol{a}_{h}\left(\left(\mathcal{L}_{h}^{f}, 0\right),\left(\boldsymbol{v}_{h}^{o}, 0\right)\right)=\int_{\Omega} \boldsymbol{f} \cdot \boldsymbol{v}_{h}^{o} \mathrm{dx}, \quad \forall \boldsymbol{v}_{h}^{o} \in \boldsymbol{V}_{h}^{k, o *} .
$$

Again, well-posedness of the above linear systems easily follows from the coercivity result (??).

Lemma 3.5 (Characterization of the condensed system for (27)). Let $\left(\boldsymbol{u}_{h}, \widehat{\boldsymbol{u}}_{h}\right) \in \boldsymbol{V}_{h, 0}^{k} \times \widehat{\boldsymbol{V}}_{h, 0}^{k-1}$ be the unique solution to the HDG scheme (27). Then,

$$
\boldsymbol{u}_{h}=\boldsymbol{u}_{h}^{\partial}+\mathcal{L}_{h}\left(\boldsymbol{u}_{h}^{\partial}, \widehat{\boldsymbol{u}}_{h}\right)+\mathcal{L}_{h}^{f},
$$


and $\left(\boldsymbol{u}_{h}^{\partial}, \widehat{\boldsymbol{u}}_{h}\right) \in \boldsymbol{V}_{h, 0}^{k, \partial} \times \widehat{\boldsymbol{V}}_{h, 0}^{k-1}$ is the unique solution to the following condensed system

$$
\boldsymbol{a}_{h}\left(\left(\boldsymbol{u}_{h}^{\partial}+\mathcal{L}_{h}\left(\boldsymbol{u}_{h}^{\partial}, \widehat{\boldsymbol{u}}_{h}\right), \widehat{\boldsymbol{u}}_{h}\right),\left(\boldsymbol{v}_{h}^{\partial}+\mathcal{L}_{h}\left(\boldsymbol{v}_{h}^{\partial}, \widehat{\boldsymbol{v}}_{h}\right), \widehat{\boldsymbol{v}}_{h}\right)\right)=-\boldsymbol{a}_{h}\left(\left(\mathcal{L}_{h}^{f}, 0\right),\left(\boldsymbol{v}_{h}^{\partial}, \widehat{\boldsymbol{v}}_{h}\right)\right)+\int_{\Omega} \boldsymbol{f} \cdot \boldsymbol{v}_{h}^{\partial} \mathrm{dx}
$$

for all $\left(\boldsymbol{v}_{h}^{\partial}, \widehat{\boldsymbol{v}}_{h}\right) \in \boldsymbol{V}_{h, 0}^{k, \partial} \times \widehat{\boldsymbol{V}}_{h, 0}^{k-1}$. In particular, the reduced system (31b) is symmetric and positive definite, and

$$
\boldsymbol{a}_{h}\left(\left(\boldsymbol{v}_{h}^{\partial}+\mathcal{L}_{h}\left(\boldsymbol{v}_{h}^{\partial}, \widehat{\boldsymbol{v}}_{h}\right), \widehat{\boldsymbol{u}}_{h}\right),\left(\boldsymbol{v}_{h}^{\partial}+\mathcal{L}_{h}\left(\boldsymbol{v}_{h}^{\partial}, \widehat{\boldsymbol{v}}_{h}\right), \widehat{\boldsymbol{v}}_{h}\right)\right) \simeq\left\|\left(\boldsymbol{v}_{h}^{\partial}+\mathcal{L}_{h}\left(\boldsymbol{v}_{h}^{\partial}, \widehat{\boldsymbol{v}}_{h}\right), \widehat{\boldsymbol{v}}_{h}\right)\right\|_{1, h}^{2},
$$

for all $\left(\boldsymbol{v}_{h}^{\partial}, \widehat{\boldsymbol{v}}_{h}\right) \in \boldsymbol{V}_{h, 0}^{k, \partial} \times \widehat{\boldsymbol{V}}_{h, 0}^{k-1}$.

Proof. See the proof of Lemma 3.1 .

3.3.3. The auxiliary space preconditioner. Now, we follow Remark 2.1 to construct the auxiliary space perconditioner for the reduced HDG system (12b).

(i) We denote the following $L^{2}$-like inner product on the compound global space $\boldsymbol{V}_{h, 0}^{k, \partial} \times \widehat{\boldsymbol{V}}_{h, 0}^{k-1}$ :

$$
\left(\left(\boldsymbol{u}_{h}^{\partial}, \widehat{\boldsymbol{u}}_{h}\right),\left(\boldsymbol{v}_{h}^{\partial}, \widehat{\boldsymbol{v}}_{h}\right)\right)_{0, h}:=\left(1+\tau h^{2}\right) \int_{\Omega}\left(\boldsymbol{u}_{h}^{\partial} \cdot \boldsymbol{v}_{h}^{\partial}\right) \mathrm{dx}+\sum_{F \in \mathcal{E}_{h}} h \int_{F} \operatorname{tang}\left(\widehat{\boldsymbol{u}}_{h}\right) \cdot \operatorname{tang}\left(\widehat{\boldsymbol{v}}_{h}\right) \mathrm{ds}
$$

whose induced norm is denoted as $\left\|\left(\boldsymbol{v}_{h}^{\partial}, \widehat{\boldsymbol{v}}_{h}\right)\right\|_{0, h}^{2}:=\left(\left(\boldsymbol{v}_{h}^{\partial}, \widehat{\boldsymbol{v}}_{h}\right),\left(\boldsymbol{v}_{h}^{\partial}, \widehat{\boldsymbol{v}}_{h}\right)\right)_{0, h}$. We define the condensed HDG operator $\boldsymbol{A}_{h}: \boldsymbol{V}_{h, 0}^{k, \partial} \times \widehat{\boldsymbol{V}}_{h, 0}^{k-1} \rightarrow \boldsymbol{V}_{h, 0}^{k, \partial} \times \widehat{\boldsymbol{V}}_{h, 0}^{k-1}$ as

$$
\left(\boldsymbol{A}_{h}\left(\boldsymbol{u}_{h}^{\partial}, \widehat{\boldsymbol{u}}_{h}\right),\left(\boldsymbol{v}_{h}^{\partial}, \widehat{\boldsymbol{v}}_{h}\right)\right)_{0, h}:=\boldsymbol{a}_{h}\left(\left(\boldsymbol{u}_{h}^{\partial}+\mathcal{L}_{h}\left(\boldsymbol{u}_{h}^{\partial}, \widehat{\boldsymbol{u}}_{h}\right), \widehat{\boldsymbol{u}}_{h}\right),\left(\boldsymbol{v}_{h}^{\partial}+\mathcal{L}_{h}\left(\boldsymbol{v}_{h}^{\partial}, \widehat{\boldsymbol{v}}_{h}\right), \widehat{\boldsymbol{v}}_{h}\right) .\right)
$$

The following result gives an estimation of the spectral radius of $\boldsymbol{A}_{h}$ and its condition number.

Lemma 3.6 (Spectral radius and condition number). Let $\boldsymbol{A}_{h}$ be the operator given in (33). Then, there holds $\rho_{\boldsymbol{A}_{h}} \simeq h^{-2}$ and $\kappa\left(\boldsymbol{A}_{h}\right) \lesssim \frac{h^{-2}}{\min \left\{\frac{\tau+1}{1+\tau h^{2}}, h^{-2}\right\}}$.

Proof. The proof is similar to that for Lemma 3.2. Here we only sketch the main steps.

A simple scaling argument implies that

$$
\left\|\boldsymbol{u}_{h}^{\partial}\right\|_{\Omega}^{2} \simeq \sum_{F \in \mathcal{E}_{h}} h\left\|\boldsymbol{u}_{h}^{\partial} \cdot \boldsymbol{n}\right\|_{F}^{2}, \quad \forall \boldsymbol{u}_{h}^{\partial} \in \boldsymbol{V}_{h, 0}^{k, \partial}
$$

To simplify notation, we denote

$$
\boldsymbol{u}_{h}^{+}:=\boldsymbol{u}_{h}^{\partial}+\mathcal{L}_{h}\left(\boldsymbol{u}_{h}^{\partial}, \widehat{\boldsymbol{u}}_{h}\right) .
$$

The Cauchy-Schwarz inequality implies that

$$
\left\|\left(\boldsymbol{u}_{h}^{\partial}, \widehat{\boldsymbol{u}}_{h}\right)\right\|_{\boldsymbol{A}_{h}} \simeq\left\|\left(\boldsymbol{u}_{h}^{+}, \widehat{\boldsymbol{u}}_{h}\right)\right\|_{1, h} \lesssim\left\|\left(\boldsymbol{u}_{h}^{\partial}, \widehat{\boldsymbol{u}}_{h}\right)\right\|_{1, h} \lesssim h^{-1}\left\|\left(\boldsymbol{u}_{h}^{\partial}, \widehat{\boldsymbol{u}}_{h}\right)\right\|_{0, h},
$$

where the last step involves the inverse inequality and triangle inequality. Hence, $\rho_{\boldsymbol{A}_{h}} \lesssim h^{-2}$.

To prove the condition number estimate, we again use the following discrete Poincaré inequality

$$
\left\|\boldsymbol{u}_{h}^{+}\right\|_{\Omega} \lesssim\left\|\left(\boldsymbol{u}_{h}^{+}, \widehat{\boldsymbol{u}}_{h}\right)\right\|_{1, h}
$$


Then,

$$
\begin{aligned}
\left\|\left(\boldsymbol{u}_{h}^{+}, \widehat{\boldsymbol{u}}_{h}\right)\right\|_{1, h}^{2} & \gtrsim(\tau+1)\left\|\boldsymbol{u}_{h}^{+}\right\|_{\Omega}^{2}+\sum_{F \in \mathcal{E}_{h}} \frac{1}{h}\left\|\operatorname{tang}\left(\boldsymbol{u}_{h}^{+}-\widehat{\boldsymbol{u}}_{h}\right)\right\|_{F}^{2} \\
& \gtrsim \sum_{F \in \mathcal{E}_{h}}\left((\tau+1)\left(h\left\|\boldsymbol{u}_{h}^{\partial} \cdot \boldsymbol{n}\right\|_{F}^{2}+h\left\|\operatorname{tang}\left(\boldsymbol{u}_{h}^{+}\right)\right\|_{F}^{2}\right)+\frac{1}{h}\left\|\operatorname{tang}\left(\boldsymbol{u}_{h}^{+}-\widehat{\boldsymbol{u}}_{h}\right)\right\|_{F}^{2}\right) \\
& \gtrsim(\tau+1)\left\|\boldsymbol{u}_{h}^{\partial}\right\|_{\Omega}^{2}+\min \left\{\tau+1, h^{-2}\right\} \sum_{F \in \mathcal{E}_{h}} h\left\|\operatorname{tang}\left(\widehat{\boldsymbol{u}}_{h}\right)\right\|_{F}^{2} \\
& \gtrsim \min \left\{\frac{\tau+1}{1+\tau h^{2}}, h^{-2}\right\}\left\|\left(\boldsymbol{u}_{h}^{\partial}, \widehat{\boldsymbol{u}}_{h}\right)\right\|_{0, h},
\end{aligned}
$$

where in the second step we used the inverse inequality and the fact that $\boldsymbol{u}_{h}^{+} \cdot \boldsymbol{n}=\boldsymbol{u}_{h}^{\partial} \cdot \boldsymbol{n}$. This implies $\lambda_{\boldsymbol{A}_{h}}^{\min } \gtrsim \min \left\{\frac{\tau+1}{1+\tau h^{2}}, h^{-2}\right\}$, hence

$$
\kappa\left(\boldsymbol{A}_{h}\right)=\frac{\rho_{\boldsymbol{A}_{h}}}{\lambda_{\boldsymbol{A}_{h}}^{\min }} \lesssim \frac{h^{-2}}{\min \left\{\frac{\tau+1}{1+\tau h^{2}}, h^{-2}\right\}} .
$$

Finally, the lower bound on the spectral radius $h^{-2} \lesssim \rho_{\boldsymbol{A}_{h}}$ following from the following estimate

$$
\left\|\left(\phi_{h}^{\partial}, \widehat{\phi}_{h}\right)\right\|_{\boldsymbol{A}_{h}}^{2} \simeq h^{-2}\left\|\left(\phi_{h}^{\partial_{h}}, \widehat{\phi}_{h}\right)\right\|_{0, h}^{2}
$$

for any pair of local bases functions $\left(\boldsymbol{\phi}_{h}^{\partial}, \widehat{\boldsymbol{\phi}}_{h}\right) \in \boldsymbol{V}_{h, 0}^{k, \partial} \times \widehat{\boldsymbol{V}}_{h, 0}^{k-1}$, which is obtained from norm equivalence of finite dimensional spaces and the scaling argument.

Remark 3.3 (The case with a large reaction parameter). When the reaction parameter $\tau \gtrsim h^{-2}$, Lemma 3.6 implies that $\kappa\left(\boldsymbol{A}_{h}\right) \simeq 1$. In this case, a simple Jacobi preconditioner is again an optimal one for $\boldsymbol{A}_{h}$.

(ii) Similar to the scalar case, we can show that the simple Jacobi smoother satisfies (2a)), and in practical implementation, we prefer to use a block symmetric Gauss-Seidel smoother to improve its efficiency.

Lemma 3.7 (Spectum of the Jacobi preconditioner). Let $\boldsymbol{D}_{h}$ be the diagonal component of $\boldsymbol{A}_{h}$. Then, the following equivalence holds for the Jacobi smoother $\boldsymbol{R}_{h}=\boldsymbol{D}_{h}^{-1}$ :

$$
\left(\boldsymbol{R}_{h}\left(\boldsymbol{u}_{h}^{\partial}, \widehat{\boldsymbol{u}}_{h}\right),\left(\boldsymbol{u}_{h}^{\partial}, \widehat{\boldsymbol{u}}_{h}\right)\right)_{0, h} \simeq \rho_{\boldsymbol{A}_{h}}^{-1}\left\|\left(\boldsymbol{u}_{h}^{\partial}, \widehat{\boldsymbol{u}}_{h}\right)\right\|_{0, h}^{2} .
$$

Proof. See the proof of Lemma 3.2.

(iii) The auxiliary space under consideration is the (vectorial) continuous piecewise linear finite element space $\mathcal{V}_{h, 0}^{1}$ in (4f) . The associated bilinear form $\boldsymbol{a}_{h, 0}$ and the linear operator $\boldsymbol{A}_{h, 0}$ are given as follows:

$$
\left[\boldsymbol{A}_{h, 0} \boldsymbol{u}_{0}, \boldsymbol{v}_{0}\right]=\boldsymbol{a}_{h, 0}\left(\boldsymbol{u}_{0}, \boldsymbol{v}_{0}\right):=\int_{\Omega}\left(\nabla \boldsymbol{u}_{0}: \nabla \boldsymbol{v}_{0}+\tau \boldsymbol{u}_{0} \cdot \boldsymbol{v}_{0}\right) \mathrm{dx}, \quad \forall \boldsymbol{u}_{0}, \boldsymbol{v}_{0} \in \mathcal{V}_{h, 0}^{1},
$$

where $[\cdot, \cdot]$ is the usual $L^{2}$-norm on $\mathcal{V}_{h, 0}^{1}$. A robust preconditioner $\boldsymbol{B}_{h, 0}$ for the operator $\boldsymbol{A}_{h, 0}$ can be readily obtained from a vector version of that for the scalar case as the operator (36) is decoupled for each component. Here we again take $\boldsymbol{B}_{h, 0}$ to be hypre's BoomerAMG preconditioner [10, 11]. 
(iv) We take the operator $\underline{\boldsymbol{\Pi}_{h}}=\left(\boldsymbol{\Pi}_{h}^{\partial}, \widehat{\boldsymbol{\Pi}}_{h}\right): \mathcal{V}_{h, 0}^{1} \rightarrow \boldsymbol{V}_{h, 0}^{k, \partial} \times \widehat{\boldsymbol{V}}_{h, 0}^{k-1}$ to be the following projection:

$$
\begin{aligned}
& \sum_{F \in \mathcal{E}_{h}} \int_{F}\left(\boldsymbol{\Pi}_{h}^{\partial} \boldsymbol{u}_{o} \cdot \boldsymbol{n}\right)\left(\boldsymbol{v}_{h}^{\partial} \cdot \boldsymbol{n}\right) \mathrm{ds}=\sum_{F \in \mathcal{E}_{h}} \int_{F}\left(\boldsymbol{u}_{o} \cdot \boldsymbol{n}\right)\left(\boldsymbol{v}_{h}^{\partial} \cdot \boldsymbol{n}\right) \mathrm{ds}, \forall \boldsymbol{v}_{h}^{\partial} \in \boldsymbol{V}_{h, 0}^{k, \partial}, \\
& \sum_{F \in \mathcal{E}_{h}} \int_{F} \operatorname{tang}\left(\widehat{\boldsymbol{\Pi}}_{h} \boldsymbol{u}_{o}\right) \cdot \operatorname{tang}\left(\widehat{\boldsymbol{v}}_{h}\right) \mathrm{ds}=\sum_{F \in \mathcal{E}_{h}} \int_{F} \operatorname{tang}\left(\boldsymbol{u}_{o}\right) \cdot \operatorname{tang}\left(\widehat{\boldsymbol{v}}_{h}\right) \mathrm{ds}, \quad \forall \widehat{\boldsymbol{v}}_{h} \in \widehat{\boldsymbol{V}}_{h, 0}^{k-1},
\end{aligned}
$$

and the operator $\boldsymbol{P}_{h}: \boldsymbol{V}_{h, 0}^{k, \partial} \times \widehat{\boldsymbol{V}}_{h, 0}^{k-1} \rightarrow \mathcal{V}_{h, 0}^{1}$ to be

$$
\boldsymbol{P}_{h}\left(\boldsymbol{u}_{h}^{\partial}, \widehat{\boldsymbol{u}}_{h}\right)=S_{1}\left(\boldsymbol{u}_{h}^{+}\right)
$$

where recall that $\boldsymbol{u}_{h}^{+}:=\boldsymbol{u}_{h}^{\partial}+\mathcal{L}_{h}\left(\boldsymbol{u}_{h}^{\partial}, \widehat{\boldsymbol{u}}_{h}\right)$ and $S_{1}$ is the simple nodal average given in (20).

The following result shows that $\underline{\boldsymbol{\Pi}}_{h}$ and $\boldsymbol{P}_{h}$ satisfies both the stability and approximation properties needed to apply the ASP theory, Theorem 2.1 .

Lemma 3.8 (Properties of $\underline{\boldsymbol{\Pi}_{h}}$ and $\boldsymbol{P}_{h}$ ). Let the operator $\underline{\boldsymbol{\Pi}_{h}}$ be given in (37) and $\boldsymbol{P}_{h}$ be given in (38). Then the following inequalities holds

$$
\begin{aligned}
\left\|\underline{\boldsymbol{\Pi}_{h}} \boldsymbol{u}_{0}\right\|_{\boldsymbol{A}_{h}} & \lesssim\left\|\boldsymbol{u}_{0}\right\|_{\boldsymbol{A}_{h, 0}}, \\
\left\|\boldsymbol{P}_{h}\left(\boldsymbol{u}_{h}^{\partial}, \widehat{\boldsymbol{u}}_{h}\right)\right\|_{\boldsymbol{A}_{h, 0}} & \lesssim\left\|\left(\boldsymbol{u}_{h}^{\partial}, \widehat{\boldsymbol{u}}_{h}\right)\right\|_{\boldsymbol{A}_{h}}, \\
\left\|\left(\boldsymbol{u}_{h}^{\partial}, \widehat{\boldsymbol{u}}_{h}\right)-\underline{\boldsymbol{\Pi}_{h}} \boldsymbol{P}_{h}\left(\boldsymbol{u}_{h}^{\partial}, \widehat{\boldsymbol{u}}_{h}\right)\right\|_{0, h} & \lesssim \rho_{\boldsymbol{A}_{h}}^{-1 / 2}\left\|\left(\boldsymbol{u}_{h}^{\partial}, \widehat{\boldsymbol{u}}_{h}\right)\right\|_{\boldsymbol{A}_{h}} .
\end{aligned}
$$

Proof. The proof is similar to that for Lemma 3.4. Here we only sketch the main steps.

Since $\mathcal{V}_{h, 0}^{1} \subset \boldsymbol{V}_{h, 0}^{k, \partial}$, we have $\boldsymbol{\Pi}_{h}^{\partial} \boldsymbol{u}_{0}=\boldsymbol{u}_{0}$ for any $\boldsymbol{u}_{0} \in \mathcal{V}_{h, 0}^{1}$. Hence,

$$
\left\|\underline{\boldsymbol{\Pi}_{h}} \boldsymbol{u}_{0}-\left(\boldsymbol{u}_{0}, \boldsymbol{u}_{0}\right)\right\|_{0, h}=\left\|\left(0, \widehat{\boldsymbol{\Pi}}_{h} \boldsymbol{u}_{0}-\boldsymbol{u}_{0}\right)\right\|_{0, h} \lesssim h\left\|\nabla \boldsymbol{u}_{0}\right\|_{\Omega} .
$$

Next, we can prove

$$
\left\|\left(\boldsymbol{u}_{0}, \boldsymbol{u}_{0}\right)\right\|_{\boldsymbol{A}_{h}} \lesssim\left\|\boldsymbol{u}_{0}\right\|_{\boldsymbol{A}_{h, 0}}, \quad \forall \boldsymbol{u}_{0} \in \mathcal{V}_{h, 0}^{1} .
$$

Then the inequality (39a) follows from the above two estimates and $\rho_{\boldsymbol{A}_{h}} \simeq h^{-2}$.

To prove the inequality (39b), we again use the following result for the average operator $S_{1}$

$$
\left\|\boldsymbol{P}_{h}\left(\boldsymbol{u}_{h}^{\partial}, \widehat{\boldsymbol{u}}_{h}\right)-\boldsymbol{u}_{h}^{+}\right\|_{\Omega}^{2} \lesssim h^{2}\left\|\nabla \boldsymbol{u}_{h}^{+}\right\|_{\mathcal{T}_{h}}^{2}+\left\|\llbracket \boldsymbol{u}_{h}^{+} \rrbracket\right\|_{0, h}^{2},
$$

which implies that

$$
\left\|\boldsymbol{P}_{h}\left(\boldsymbol{u}_{h}^{\partial}, \widehat{\boldsymbol{u}}_{h}\right)-\boldsymbol{u}_{h}^{+}\right\|_{\Omega} \lesssim \min \left\{\left\|\boldsymbol{u}_{h}^{+}\right\|_{\Omega}, h\left\|\left(\boldsymbol{u}_{h}^{\partial}, \widehat{\boldsymbol{u}}_{h}\right)\right\|_{\boldsymbol{A}_{h}}\right\}
$$

Hence, we have

$$
\left\|\boldsymbol{P}_{h}\left(\boldsymbol{u}_{h}^{\partial}, \widehat{\boldsymbol{u}}_{h}\right)\right\|_{\boldsymbol{A}_{h, 0}} \lesssim\left\|\boldsymbol{P}_{h}\left(\boldsymbol{u}_{h}^{\partial}, \widehat{\boldsymbol{u}}_{h}\right)-\boldsymbol{u}_{h}^{+}\right\|_{\boldsymbol{A}_{h, 0}}+\left\|\boldsymbol{u}_{h}^{+}\right\|_{\boldsymbol{A}_{h, 0}} \lesssim\left\|\left(\boldsymbol{u}_{h}^{\partial}, \widehat{\boldsymbol{u}}_{h}\right)\right\|_{\boldsymbol{A}_{h}}
$$

Finally, let us prove the approximation property (21c). The further simply notation, we denote $\boldsymbol{P}_{h}^{+}:=\boldsymbol{P}_{h}\left(\boldsymbol{u}_{h}^{\partial}, \widehat{\boldsymbol{u}}_{h}\right)$. Then, there holds

$$
\begin{aligned}
\left\|\left(\boldsymbol{u}_{h}^{\partial}, \widehat{\boldsymbol{u}}_{h}\right)-\underline{\boldsymbol{\Pi}_{h}} \boldsymbol{P}_{h}^{+}\right\|_{0, h}^{2} & \lesssim\left\|\left(\boldsymbol{u}_{h}^{\partial}-\boldsymbol{P}_{h}^{+}, \widehat{\boldsymbol{u}}_{h}-\boldsymbol{u}_{h}^{+}\right)\right\|_{0, h}^{2}+\left\|\left(0, \boldsymbol{u}_{h}^{+}-\boldsymbol{P}_{h}^{+}\right)\right\|_{0, h}^{2}+\left\|\left(\boldsymbol{P}_{h}^{+}, \boldsymbol{P}_{h}^{+}\right)-\underline{\boldsymbol{\Pi}_{h}} \boldsymbol{P}_{h}^{+}\right\|_{0, h}^{2} \\
& \lesssim\left(1+\tau h^{2}\right)\left\|\boldsymbol{u}_{h}^{\partial}-\boldsymbol{P}_{h}^{+}\right\|_{\Omega}^{2}+h^{2}\left\|\left(\boldsymbol{u}_{h}^{+}, \widehat{\boldsymbol{u}}_{h}\right)\right\|_{\boldsymbol{A}_{h}}^{2}+\left\|\boldsymbol{u}_{h}^{+}-\boldsymbol{P}_{h}^{+}\right\|_{\Omega}^{2}+h^{2}\left\|\nabla \boldsymbol{P}_{h}^{+}\right\|_{\Omega}^{2}, \\
& \lesssim\left(1+\tau h^{2}\right)\left\|\boldsymbol{u}_{h}^{+}-\boldsymbol{P}_{h}^{+}\right\|_{\Omega}^{2}+h^{2}\left\|\left(\boldsymbol{u}_{h}^{+}, \widehat{\boldsymbol{u}}_{h}\right)\right\|_{\boldsymbol{A}_{h}}^{2} \lesssim h^{2}\left\|\left(\boldsymbol{u}_{h}^{+}, \widehat{\boldsymbol{u}}_{h}\right)\right\|_{\boldsymbol{A}_{h}}^{2},
\end{aligned}
$$

where in the third step we used the fact that

$$
\left\|\boldsymbol{u}_{h}^{\partial}-\boldsymbol{P}_{h}^{+}\right\|_{\Omega}^{2} \simeq h \sum_{F \in \mathcal{E}_{h}}\left\|\left(\boldsymbol{u}_{h}^{\partial}-\boldsymbol{P}_{h}^{+}\right) \cdot \boldsymbol{n}\right\|_{F}^{2}=h \sum_{F \in \mathcal{E}_{h}}\left\|\left(\boldsymbol{u}_{h}^{+}-\boldsymbol{P}_{h}^{+}\right) \cdot \boldsymbol{n}\right\|_{F}^{2} \lesssim\left\|\boldsymbol{u}_{h}^{+}-\boldsymbol{P}_{h}^{+}\right\|_{\Omega}^{2} .
$$


This completes the sketchy proof.

(v) Finally, we conclude the following optimality of an auxiliary space preconditioner by invoking Theorem 2.1 and applying Lemma 3.6-Lemma 3.8.

Theorem 3.2 (ASP for the condensed HDG operator (33)). Let $\boldsymbol{B}_{h}=\boldsymbol{R}_{h}+\underline{\boldsymbol{\Pi}}_{h} \boldsymbol{B}_{h, 0} \underline{\boldsymbol{\Pi}}_{h}{ }^{t}$ be the auxiliary space preconditioner for the operator $\boldsymbol{A}_{h}$ in (33) with $\boldsymbol{R}_{h}$ being the Jacobi smoother for $\boldsymbol{A}_{h}, \boldsymbol{B}_{h, 0}$ being an optimal preconditioner for $\boldsymbol{A}_{h, 0}$ in $(\underline{36})$ such that $\kappa\left(\boldsymbol{B}_{h, 0} \boldsymbol{A}_{h, 0}\right) \simeq 1$, and $\underline{\boldsymbol{\Pi}}_{h}$ being the projector in (37). Then, $\kappa\left(\boldsymbol{B}_{h} \boldsymbol{A}_{h}\right) \simeq 1$.

Remark 3.4 (On reduced divergence-conforming space). All the above results still hold if we replace the divergence-conforming space $\boldsymbol{V}_{h, 0}^{k}$ in the HDG scheme (27) by the reduced divergenceconforming space $\boldsymbol{V}_{h, 0}^{k, \mathrm{cst}}$ in (4h). The only change would be a smaller local space $\boldsymbol{V}_{h}^{k, o}$ compared with the original local space $\boldsymbol{V}_{h}^{k, o *}$ for the scheme (27). While such a modification leads to a smaller set of total DOFs, it is not suggested for the HDG discretization of the current reaction-diffusion equation (26) as it leads to accuracy loss compared with the original HDG scheme (27). On the other hand, the reduced system would serve as a good preconditioner for the velocity block of a divergence-free $H D G$ discretization of incompressible flow. It can also be used to precondition a $C^{0}$-continuous interior penalty HDG scheme for the generalized biharmonic equation as we show next.

\section{4. $C^{0}$-continuous interior penalty HDG for the generalized biharmonic problem.}

3.4.1. The model and the HDG scheme. We consider the following constant-coefficient generalized biharmonic equation in a convex polygonal domain $\Omega \subset \mathbb{R}^{2}$ with a simply supported boundary condition:

$$
-\triangle^{2} \phi-\tau \triangle \phi=f \text { in } \Omega,\left.\quad \phi\right|_{\partial \Omega}=\left.\triangle \phi\right|_{\partial \Omega}=0 .
$$

Calculus identities show that

$$
\triangle^{2} \phi=\nabla \times(\nabla \cdot \nabla(\vec{\nabla} \times \phi)), \quad \triangle \phi=\nabla \times(\vec{\nabla} \times \phi),
$$

where $\vec{\nabla} \times \phi:=\left(\partial_{y} \phi,-\partial_{x} \phi\right)$ is the vectorial curl operator (rotated gradient) for a scalar field $\phi$ and $\nabla \times\left(v_{1}, v_{2}\right):=\partial_{x} v_{2}-\partial_{y} v_{1}$ is the scalar curl operator (rotated divergence) for the vector field $\left(v_{1}, v_{2}\right)$. Also, for any function $\phi$ that vanishes on the boundary $\partial \Omega$, there holds

$$
\left.\triangle \phi\right|_{\partial \Omega}=\left.\partial_{n} \partial_{n} \phi\right|_{\partial \Omega}=\left.(((\nabla \vec{\nabla} \times \phi) \boldsymbol{n}) \cdot \boldsymbol{t})\right|_{\partial \Omega}=0
$$

where $\partial_{n}$ is the directional derivative along the normal direction $\boldsymbol{n}=\left(n_{1}, n_{2}\right)$, and $\boldsymbol{t}=\left(n_{2},-n_{1}\right)$ is the tangential direction. Hence, the equation (43) is identical to the following form:

$$
-\nabla \times(\nabla \cdot \nabla(\vec{\nabla} \times \phi))-\tau \nabla \times(\vec{\nabla} \times \phi)=f \text { in } \Omega,\left.\quad \phi\right|_{\partial \Omega}=\left.(((\nabla \vec{\nabla} \times \phi) \boldsymbol{n}) \cdot \boldsymbol{t})\right|_{\partial \Omega}=0 .
$$

Next, we present a $C^{0}$-continuous interior penalty HDG (CIP-HDG) discretization of the equation (44). We didn't find in the literature on publications on CIP-HDG formulations for the generalized biharmonic equation, but it can be easily adapted from a known CIP-DG scheme [4]. We mention that the implementation of a version of the CIP-HDG scheme for the biharmonic equation was already used in unit 2.9 of the $i$-tutorials of the NGSolve software [17.

Given a polynomial degree $k \geq 1$, the CIP-HDG scheme with projected jumps reads as follows: find $\left(\phi_{h}, \widehat{\boldsymbol{u}}_{h}\right) \in X_{h, 0}^{k+1} \times \widehat{\boldsymbol{V}}_{h}^{k-1}$ such that

$$
k_{h}\left(\left(\phi_{h}, \widehat{\boldsymbol{u}}_{h}\right),\left(\psi_{h}, \widehat{\boldsymbol{v}}_{h}\right)\right)=\int_{\Omega} f \psi_{h} \mathrm{dx}, \quad \forall\left(\psi_{h}, \widehat{\boldsymbol{v}}_{h}\right) \in X_{h, 0}^{k+1} \times \widehat{\boldsymbol{V}}_{h}^{k-1},
$$


where the bilinear form

$$
\begin{aligned}
k_{h}\left(\left(\phi_{h}, \widehat{\boldsymbol{u}}_{h}\right),\left(\psi_{h}, \widehat{\boldsymbol{v}}_{h}\right)\right):=\sum_{K \in \mathcal{T}_{h}} & \int_{K}\left(\nabla \vec{\nabla} \times \phi_{h}: \nabla \vec{\nabla} \times \psi_{h}+\tau \vec{\nabla} \times \phi_{h} \cdot \vec{\nabla} \times \psi_{h}\right) \mathrm{dx} \\
& -\int_{\partial K}\left(\nabla \vec{\nabla} \times \phi_{h}\right) \boldsymbol{n} \cdot \operatorname{tang}\left(\vec{\nabla} \times \psi_{h}-\widehat{\boldsymbol{v}}_{h}\right) \mathrm{ds} \\
& -\int_{\partial K}\left(\nabla \vec{\nabla} \times \psi_{h}\right) \boldsymbol{n} \cdot \operatorname{tang}\left(\vec{\nabla} \times \phi_{h}-\widehat{\boldsymbol{u}}_{h}\right) \mathrm{ds} \\
& +\int_{\partial K} \frac{\alpha k^{2}}{h} \operatorname{tang}\left(\boldsymbol{P}_{k-1} \vec{\nabla} \times \phi_{h}-\widehat{\boldsymbol{u}}_{h}\right) \cdot \operatorname{tang}\left(\boldsymbol{P}_{k-1} \vec{\nabla} \times \psi_{h}-\widehat{\boldsymbol{v}}_{h}\right) \mathrm{ds},
\end{aligned}
$$

where $\widehat{\boldsymbol{u}}_{h} \in \widehat{\boldsymbol{V}}_{h}^{k-1}$ is the approximation of the tangential component of $\vec{\nabla} \times \phi_{h}$ on the mesh skeleton. Note that we do not impose any boundary constraints on the tangential facet finite element space $\widehat{\boldsymbol{V}}_{h}^{k-1}$ to respect the simply supported boundary condition in (44). Here, again, we take the stability parameter $\alpha=4$ to ensure the coercivity. In particular, the following norm equivalence holds

$$
k_{h}\left(\left(\phi_{h}, \widehat{\boldsymbol{u}}_{h}\right),\left(\phi_{h}, \widehat{\boldsymbol{u}}_{h}\right)\right) \simeq\left\|\left(\phi_{h}, \widehat{\boldsymbol{u}}_{h}\right)\right\|_{1, h}^{2},
$$

where

$$
\left\|\left(\phi_{h}, \widehat{\boldsymbol{u}}_{h}\right)\right\|_{1, h}^{2}:=\sum_{K \in \mathcal{T}_{h}}\left(\left\|\nabla \vec{\nabla} \times \phi_{h}\right\|_{K}^{2}+\tau\left\|\vec{\nabla} \times \phi_{h}\right\|_{K}^{2}+\frac{1}{h}\left\|\vec{\nabla} \times \phi_{h}-\widehat{\boldsymbol{u}}_{h}\right\|_{\partial K}^{2}\right)
$$

is a norm on $X_{h, 0}^{k+1} \times \widehat{\boldsymbol{V}}_{h}^{k-1}$. A direct comparison of the CIP-HDG bilinear form $k_{h}$ in (45) with the divergence-conforming HDG bilinear form $\boldsymbol{a}_{h}$ in (27) implies that

$$
k_{h}\left(\left(\phi_{h}, \widehat{\boldsymbol{u}}_{h}\right),\left(\psi_{h}, \widehat{\boldsymbol{v}}_{h}\right)\right)=\boldsymbol{a}_{h}\left(\left(\vec{\nabla} \times \phi_{h}, \widehat{\boldsymbol{u}}_{h}\right),\left(\vec{\nabla} \times \psi_{h}, \widehat{\boldsymbol{u}}_{h}\right)\right),
$$

for all $\left(\phi_{h}, \widehat{\boldsymbol{u}}_{h}\right),\left(\psi_{h}, \widehat{\boldsymbol{v}}_{h}\right) \in X_{h, 0}^{k+1} \times \widehat{\boldsymbol{V}}_{h}^{k-1}$.

3.4.2. The auxiliary space preconditioner. Here we directly construct the auxiliary space preconditioner for the system (45) without static condensation. The analysis with static condensation is similar the previous subsection and we omit it to avoid unnecessary repetition. In our numerical implementation, static condensation is of course activated to improve efficiency of the solver. The key idea is to use the divergence-conforming HDG scheme (27) for reaction-diffusion to precondition the scheme (45), see the original idea in 3. The obtained preconditioner is actually a fictitious space preconditioner [16] as no smoother is involved in the preconditioner.

We define the HDG operator $K_{h}: X_{h, 0}^{k+1} \times \widehat{\boldsymbol{V}}_{h}^{k-1} \rightarrow X_{h, 0}^{k+1} \times \widehat{\boldsymbol{V}}_{h}^{k-1}$ as

$$
\left[K_{h}\left(\phi_{h}, \widehat{\boldsymbol{u}}_{h}\right),\left(\psi_{h}, \widehat{\boldsymbol{v}}_{h}\right)\right]_{0, h}:=k_{h}\left(\left(\phi_{h}, \widehat{\boldsymbol{u}}_{h}\right),\left(\psi_{h}, \widehat{\boldsymbol{v}}_{h}\right)\right),
$$

where $[\cdot, \cdot]_{0, h}$ is an inner product on $X_{h, 0}^{k+1} \times \widehat{\boldsymbol{V}}_{h}^{k-1}$. The auxiliary space to be considered is $\boldsymbol{V}_{h, 0}^{k, \text { cst }} \times$ $\widehat{\boldsymbol{V}}_{h}^{k-1}$. With an abuse of notation, we denote $\boldsymbol{A}_{h}: \boldsymbol{V}_{h, 0}^{k, \text { cst }} \times \widehat{\boldsymbol{V}}_{h}^{k-1} \rightarrow \boldsymbol{V}_{h, 0}^{k, \text { cst }} \times \widehat{\boldsymbol{V}}_{h}^{k-1}$ as the linear operator associated with the bilinear form $\boldsymbol{a}_{h}$ in (27):

$$
\left(\boldsymbol{A}_{h}\left(\boldsymbol{u}_{h}, \widehat{\boldsymbol{u}}_{h}\right),\left(\boldsymbol{v}_{h}, \widehat{\boldsymbol{v}}_{h}\right)\right)_{0, h}:=\boldsymbol{a}_{h}\left(\left(\boldsymbol{u}_{h}, \widehat{\boldsymbol{u}}_{h}\right),\left(\boldsymbol{v}_{h}, \widehat{\boldsymbol{v}}_{h}\right)\right), \quad \forall\left(\boldsymbol{u}_{h}, \widehat{\boldsymbol{u}}_{h}\right),\left(\boldsymbol{v}_{h}, \widehat{\boldsymbol{v}}_{h}\right) \in \boldsymbol{V}_{h, 0}^{k, \text { cst }} \times \widehat{\boldsymbol{V}}_{h}^{k-1}
$$


An optimal preconditioner for $\boldsymbol{A}_{h}$ was already constructed in Theorem 3.2. We further define the following mapping operator $\underline{\Pi_{h}}=\left(\Pi_{h}, \widehat{\Pi}_{h}\right): \boldsymbol{V}_{h, 0}^{k, \text { cst }} \times \widehat{\boldsymbol{V}}_{h}^{k-1} \rightarrow X_{h, 0}^{k+1} \times \widehat{\boldsymbol{V}}_{h}^{k-1}$ :

$$
\begin{aligned}
\int_{\Omega}\left(\vec{\nabla} \times \Pi_{h}\left(\boldsymbol{u}_{h}, \widehat{\boldsymbol{u}}_{h}\right) \cdot \vec{\nabla} \times \psi_{h} \mathrm{dx}=\int_{\Omega}\left(\boldsymbol{u}_{h} \cdot \vec{\nabla} \times \psi_{h}\right) \mathrm{dx}, \quad \forall \psi_{h} \in X_{h, 0}^{k+1},\right. \\
\sum_{K \in \mathcal{T}_{h}} \int_{\partial K} \operatorname{tang}\left(\widehat{\Pi}_{h}\left(\boldsymbol{u}_{h}, \widehat{\boldsymbol{u}}_{h}\right)-\Pi_{h}\left(\boldsymbol{u}_{h}, \widehat{\boldsymbol{u}}_{h}\right)\right) \cdot \operatorname{tang}\left(\widehat{\boldsymbol{v}}_{h}\right) \mathrm{ds}=0, \quad \forall \widehat{\boldsymbol{v}}_{h} \in \widehat{\boldsymbol{V}}_{h}^{k-1} .
\end{aligned}
$$

Here (50b) implies that

$$
\operatorname{tang}\left(\widehat{\Pi}_{h}\left(\boldsymbol{u}_{h}, \widehat{\boldsymbol{u}}_{h}\right)\right)=\operatorname{tang}\left(\boldsymbol{P}_{k-1}\left\{\left\{\Pi_{h}\left(\boldsymbol{u}_{h}, \widehat{\boldsymbol{u}}_{h}\right)\right\}\right),\right.
$$

where $\left\{\{\mu\}\right.$ is the average operator on interior facets of a function $\mu$, and $\left.\{\mu \mu\}\right|_{\partial \Omega}=\mu$.

The main result of this subsection is now summarized below.

Theorem 3.3 (ASP for the HDG operator (48)) . Let $M_{h}=\Pi_{h} \boldsymbol{B}_{h} \Pi_{h}{ }^{t}$ be the auxiliary space preconditioner for the operator $K_{h}$ in (48) with $\underline{\Pi_{h}}$ given in (50) $\overline{\text { and }} B_{h} \bar{b}$ eing an optimal preconditioner for $A_{h}$ in (49). Then, $\kappa\left(M_{h} K_{h}\right) \simeq 1$.

Proof. Taking $\mathrm{P}_{h}: X_{h, 0}^{k+1} \times \widehat{\boldsymbol{V}}_{h}^{k-1} \rightarrow \boldsymbol{V}_{h, 0}^{k, \text { cst }} \times \widehat{\boldsymbol{V}}_{h}^{k-1}$ as the following natural inclusion:

$$
\mathrm{P}_{h}\left(\phi_{h}, \widehat{\boldsymbol{u}}_{h}\right):=\left(\vec{\nabla} \times \phi_{h}, \widehat{\boldsymbol{u}}_{h}\right), \quad \forall\left(\phi_{h}, \widehat{\boldsymbol{u}}_{h}\right) \in X_{h, 0}^{k+1} \times \widehat{\boldsymbol{V}}_{h}^{k-1} .
$$

It is trivial to show that

$$
\left\|\mathrm{P}_{h}\left(\phi_{h}, \widehat{\boldsymbol{u}}_{h}\right)\right\|_{\boldsymbol{A}_{h}}=\left\|\left(\phi_{h}, \widehat{\boldsymbol{u}}_{h}\right)\right\|_{K_{h}}
$$

and that $\mathrm{P}_{h}$ is a right inverse of $\Pi_{h}$ :

$$
\underline{\Pi_{h}} \mathrm{P}_{h}\left(\phi_{h}, \widehat{\boldsymbol{u}}_{h}\right)=\left(\phi_{h}, \widehat{\boldsymbol{u}}_{h}\right) .
$$

Hence, we only need to prove the following stability of the projector $\underline{\Pi_{h}}$ :

$$
\left\|\underline{\Pi_{h}}\left(\boldsymbol{u}_{h}, \widehat{\boldsymbol{u}}_{h}\right)\right\|_{K_{h}} \lesssim\left\|\left(\boldsymbol{u}_{h}, \widehat{\boldsymbol{u}}_{h}\right)\right\|_{\boldsymbol{A}_{h}}
$$

for the optimality of the preconditioner $M_{h}$. To simplify notation, we denote $\boldsymbol{u}_{h}^{*}:=\vec{\nabla} \times \Pi_{h}\left(\boldsymbol{u}_{h}, \widehat{\boldsymbol{u}}_{h}\right)$. Hence,

$$
\operatorname{tang}\left(\widehat{\Pi}_{h}\left(\boldsymbol{u}_{h}, \widehat{\boldsymbol{u}}_{h}\right)\right)=\operatorname{tang}\left(\boldsymbol{P}_{k-1}\left\{\left\{\boldsymbol{u}_{h}^{*}\right\}\right\}\right)
$$

by (51). We then have, by (46),

$$
\left\|\underline{\Pi_{h}}\left(\boldsymbol{u}_{h}, \widehat{\boldsymbol{u}}_{h}\right)\right\|_{K_{h}} \lesssim \sum_{K \in \mathcal{T}_{h}}\left(\left\|\nabla \boldsymbol{u}_{h}^{*}\right\|_{K}^{2}+\tau\left\|\boldsymbol{u}_{h}^{*}\right\|_{K}^{2}+\frac{1}{h}\left\|\operatorname{tang}\left(\llbracket \boldsymbol{u}_{h}^{*} \rrbracket\right)\right\|_{\partial K}^{2}\right),
$$

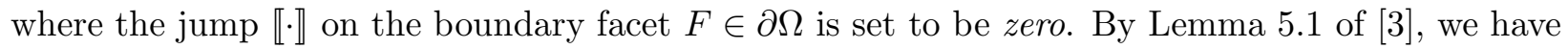

$$
\sum_{K \in \mathcal{T}_{h}}\left(\left\|\nabla\left(\boldsymbol{u}_{h}^{*}-\boldsymbol{u}_{h}\right)\right\|_{K}^{2}+\frac{1}{h}\left\|\operatorname{tang}\left(\llbracket \boldsymbol{u}_{h}^{*}-\boldsymbol{u}_{h} \rrbracket\right)\right\|_{\partial K}^{2}\right) \lesssim\left\|\nabla \cdot \boldsymbol{u}_{h}\right\|_{\Omega} .
$$

Taking $\vec{\nabla} \times \psi_{h}=\boldsymbol{u}_{h}^{*}$ in (50a) and applying the Cauchy-Schwarz inequality, we further have

$$
\left\|\boldsymbol{u}_{h}^{*}\right\|_{\Omega} \leq\left\|\boldsymbol{u}_{h}\right\|_{\Omega}
$$

Combining the above two estimates with the inequality (53) and a triangle inequality, we conclude the proof for the stability result (52). 
Remark 3.5 (On boundary conditions). Here we remark that the assumption on the simply supported boundary condition (44) is crucial to our analysis, as the result (54) no longer holds true if we have a clamped boundary condition, $\left.\phi\right|_{\partial \Omega}=\left.\partial_{n} \phi\right|_{\partial \Omega}=0$. In this case, we shall use $\widehat{\boldsymbol{V}}_{h, 0}^{k-1}$ as the facet finite element space in the CIP-HDG scheme (45) to respect the clamped boundary condition. Then, we have

$$
\left.\operatorname{tang}\left(\widehat{\Pi}_{h}\left(\boldsymbol{u}_{h}, \widehat{\boldsymbol{u}}_{h}\right)\right)\right|_{\partial \Omega}=0 \neq\left.\operatorname{tang}\left(\boldsymbol{P}_{k-1} \Pi_{h}\left(\boldsymbol{u}_{h}, \widehat{\boldsymbol{u}}_{h}\right)\right)\right|_{\partial \Omega}
$$

due to the homogeneous boundary condition on $\widehat{\boldsymbol{V}}_{h, 0}^{k-1}$. Hence the right hand side of the inequality (53) shall also include the boundary contribution $\sum_{F \in \mathcal{E}_{h}^{\partial}} \frac{1}{h}\left\|\operatorname{tang}\left(\boldsymbol{u}_{h}^{*}\right)\right\|_{F}^{2}$, which, however, can not be controlled by $\left\|\left(\boldsymbol{u}_{h}, \widehat{\boldsymbol{u}}_{h}\right)\right\|_{\boldsymbol{A}_{h}}^{2}$, see the proof of [3, Lemma 5.1].

Our numerical results also indicate that Theorem 3.3 fails to hold for the clamped boundary condition case as the preconditioner is no longer optimal with respect to the mesh size $h$. This is to be contrasted to the results in the previous two subsections, where we considered the homogeneous Dirichlet boundary condition only for the purpose of simplicity of the presentation as the main results therein still hold if we replace the Dirichlet boundary condition by other standard boundary conditions.

As can be seen clearly from the analysis of Theorem 3.3, we only need to prove stability of the projector (52) for the optimality of the associated preconditioner. However, we are not able to construct an easy-to-compute projector that achieve this. In a forthcoming paper, we will address the preconditioning issue of (45) with a clamped boundary condition using a different technique.

Remark 3.6 (On computational cost of the preconditioner $M_{h}$ ). The main computation cost to computer the projector $\Pi_{h}$ is the Poisson solver (50a) on the space $X_{h, 0}^{k+1}$. If we take $\boldsymbol{B}_{h}$ to be the ASP developed in Theorem 3.2 for $\boldsymbol{A}_{h}$, then it involves a Poisson-like solver for the operator $\boldsymbol{A}_{h, 0}$ (36) on the space $\mathcal{V}_{h, 0}^{1}$, which is equivalent to two scalar Poisson-like solvers. Hence, an application of the preconditioner $M_{h}$ would involve two Poisson solvers on the high-order $H^{1}$-conforming space $X_{h, 0}^{k+1}$ (one for $\underline{\Pi_{h}}$ and one for ${\underline{\Pi_{h}}}^{t}$ ) and two Poisson-like solver on the low-order $H^{1}$-conforming space $\mathcal{W}_{h, 0}^{1}$.

On the other hand, if $\tau \geq h^{-2}$, we can take $\boldsymbol{B}_{h}$ as the simple Jacobi preconditioner for $\boldsymbol{A}_{h}$ which is optimal due to Lemma 3.6. In this case, the cost of an application of the preconditioner $M_{h}$ would be two Poisson solvers on the high-order $H^{1}$-conforming space $X_{h, 0}^{k+1}$.

Remark 3.7 (Equivalence of CIP-HDG for biharmonic equation with divergence-free HDG for Stokes flow). It is well-known that the CIP-DG scheme [4] for biharmonic equation is equivalent to a divergence-free $D G$ scheme for Stokes flow, see [12]. The same conclusion can also be made for the current CIP-HDG scheme (45) and the divergence-free HDG scheme for a generalized Stokes flow [13, Chapter 2]. Hence, we also obtained a robust preconditioner for the divergence-free HDG scheme for the generalized Stokes flow with a slip boundary condition.

\section{Numerical ReSUlts}

In this section we perform some numerical experiments for the preconditioners discussed in the previous section. All numerical examples are performed using the NGSolve software [17. Full code examples are available at www.github.com/gridfunction/asp-hdg/.

For the symmetric interior penalty HDG scheme (7), we take the domain to be a unit cube $\Omega^{3 \mathrm{~d}}=[0,1]^{3}$ with two subdomains $\Omega_{1}^{3 \mathrm{~d}}=[0.25,0.5]^{3} \cup[0.5,0.75]^{3}$ and $\Omega_{2}^{3 \mathrm{~d}}=\Omega^{3 \mathrm{~d}} \backslash \Omega_{1}^{3 \mathrm{~d}}$; for the divergence-conforming HDG scheme (27) and the CIP-HDG scheme (45), we take the domain to be a unit square $\Omega^{2 \mathrm{~d}}=[0,1]^{2}$ with two subdomains $\Omega_{1}^{2 \mathrm{~d}}=[0.25,0.5]^{2} \cup[0.5,0.75]^{2}$ and $\Omega_{2}^{2 \mathrm{~d}}=\Omega^{2 \mathrm{~d}} \backslash \Omega_{1}^{2 \mathrm{~d}}$; In all the examples, we take the parameter $\tau=\tau_{1}$ on subdomain $\Omega_{1}$ and $\tau=\tau_{2}$ on subdomain $\Omega_{2}$ with the constants $\tau_{1}, \tau_{2} \in\left\{1,10^{4}\right\}$. We take the right hand side $f=1$, and report the number of 
PCG iterations need to reduce the relative residual by a factor of $10^{-10}$. We use a regular simplicial mesh by first dividing the domain into uniform $N \times N$ squares in $2 \mathrm{D}$ or $N \times N \times N$ cubes in 3D, then split the square into two triangles or the cube into 6 tetrahedra. $N=8$ for the coarsest mesh under consideration. We take polynomial degree $k \in\{1,4,7,10\}$.

For the scalar and vectorial reaction diffusion problems in (7) and (27), we focus on the ASP in Theorems 3.13 .2 with a point Jacobi smoother, abbreviated as JAC-ASP, and the ASP with a block symmetric Gauss-Seidel smoother using an overlapping facet-patch based block, abbreviated as BGS-ASP. For the block preconditioner, the total number of blocks is the total number of (interior) facets on the mesh, with each block being associated with a facet $F$ that contains the global DOFs which are adjacent to $F$, i.e., it contains the collection of all global DOFs on the facet $F^{\prime}$ such that $F^{\prime}$ and $F$ are shared by a common simplicial element $K$. For the generalized biharmonic problem (45), we focus on the ASP in Theorem 3.3 with a either direct solver $\boldsymbol{B}_{h}$, which is abbreviate as ASP-DIR, or the ASP with a block symmetric Gauss-Seidel smoother in Theorem 3.2, which is abbreviated as ASP-ASP.

The iteration counts for the HDG scheme (17) are recorded in Table 1, those for the divergenceconforming HDG scheme (27) are recorded in Table 2, and for the CIP-HDG scheme (45) in Table 3 for the simply supported boundary case and in Table 4 for the clamped boundary case.

From Table 1, we observe that the iteration counts are essentially independent of the mesh size $h=1 / N$ for a fixed polynomial degree and reaction parameter $\tau$ for both JAC-ASP and BGS-ASP, which verify the result of Theorem 3.1. The iteration counts are also quite robust with respect to the variations in $\tau$, where the case $\tau_{1}=\tau_{2}=10^{4}$ records the smallest number of iterations for all tests. Due to a large reaction coefficient in this case, the HDG operator (14) itself is well-conditioned, see Remark 3.1. Moreover, the growth of iteration counts on polynomial degree JAC-ASP seems to be linear, while that for BGS-ASP is very mild where they are almost independent of the polynomial degree.

The results in Table 2 for the divergence-conforming HDG scheme (27) are similar to those for Table 1, which numerically verify the result of Theorem 3.2 .

Finally, we also obtain similar results in Table 3 for the simply supported boundary condition case, where we notice that switching from a direct solver in ASP-DIR for the auxiliary operator $\boldsymbol{A}_{h}$ to the ASP in ASP-ASP only leads to a small increase on the iteration counts. On the other hand, the results in Table 4 for the clamped boundary condition case show a mesh dependency for iterations counts, especially for the case with $\tau_{1}=\tau_{2}=1$. This suggest that the proposed preconditioner is not robust for the clamped boundary condition case.

\begin{tabular}{cc|cc|cc|cc|cc}
\hline$k$ & $N$ & \multicolumn{2}{c|}{$\tau_{1}=1, \tau_{2}=1$} & \multicolumn{2}{c}{$\tau_{1}=1, \tau_{2}=10^{4}$} & $\tau_{1}=10^{4}, \tau_{2}=1$ & \multicolumn{2}{c}{$\tau_{1}=10^{4}, \tau_{2}=10^{4}$} \\
\hline & & JAC-ASP & BGS-ASP & JAC-ASP & BGS-ASP & JAC-ASP & BGS-ASP & JAC-ASP & BGS-ASP \\
\hline & 8 & 54 & 15 & 57 & 17 & 34 & 11 & 12 & 8 \\
1 & 16 & 55 & 17 & 58 & 19 & 50 & 16 & 19 & 10 \\
& 32 & 53 & 17 & 59 & 19 & 58 & 16 & 41 & 11 \\
& 64 & 51 & 16 & 55 & 17 & 55 & 17 & 46 & 12 \\
\hline \multirow{4}{*}{4} & 8 & 94 & 20 & 99 & 21 & 78 & 16 & 43 & 12 \\
& 16 & 91 & 20 & 98 & 21 & 91 & 19 & 58 & 13 \\
& 32 & 86 & 20 & 95 & 20 & 93 & 19 & 69 & 15 \\
\hline \multirow{2}{*}{7} & 8 & 142 & 23 & 149 & 24 & 124 & 19 & 75 & 15 \\
& 16 & 137 & 23 & 148 & 23 & 138 & 21 & 97 & 17 \\
\hline
\end{tabular}

TABLE 1. 3D scalar reaction-diffusion. Number of PCG iteration counts for the interior penalty HDG scheme (7). 


\begin{tabular}{|c|c|c|c|c|c|c|c|c|c|}
\hline \multirow[t]{2}{*}{$k$} & \multirow[t]{2}{*}{$\bar{N}$} & \multicolumn{2}{|c|}{$\tau_{1}=1, \tau_{2}=1$} & \multicolumn{2}{|c|}{$\tau_{1}=1, \tau_{2}=10^{4}$} & \multicolumn{2}{|c|}{$\tau_{1}=10^{4}, \tau_{2}=1$} & \multicolumn{2}{|c|}{$\tau_{1}=10^{4}, \tau_{2}=10^{4}$} \\
\hline & & JAC-ASP & BGS-ASP & JAC-ASP & BGS-ASP & JAC-ASP & BGS-ASP & JAC-ASP & BGS-ASP \\
\hline \multirow{4}{*}{1} & 8 & 62 & 15 & 67 & 15 & 52 & 16 & 34 & 10 \\
\hline & 16 & 66 & 16 & 76 & 17 & 62 & 19 & 39 & 9 \\
\hline & 32 & 64 & 15 & 76 & 17 & 69 & 18 & 46 & 8 \\
\hline & 64 & 61 & 15 & 74 & 17 & 74 & 17 & 56 & 11 \\
\hline \multirow{4}{*}{4} & 8 & 123 & 19 & 127 & 20 & 107 & 18 & 72 & 13 \\
\hline & 16 & 121 & 19 & 131 & 20 & 128 & 22 & 95 & 15 \\
\hline & 32 & 116 & 19 & 128 & 20 & 127 & 22 & 109 & 16 \\
\hline & 64 & 110 & 19 & 123 & 20 & 124 & 20 & 113 & 17 \\
\hline \multirow{4}{*}{7} & 8 & 157 & 21 & 170 & 22 & 151 & 21 & 106 & 17 \\
\hline & 16 & 157 & 21 & 172 & 23 & 167 & 26 & 132 & 18 \\
\hline & 32 & 150 & 21 & 169 & 23 & 165 & 26 & 148 & 18 \\
\hline & 64 & 143 & 20 & 163 & 22 & 162 & 23 & 151 & 19 \\
\hline \multirow{4}{*}{10} & 8 & 207 & 24 & 215 & 25 & 203 & 24 & 139 & 18 \\
\hline & 16 & 200 & 24 & 216 & 25 & 216 & 27 & 168 & 20 \\
\hline & 32 & 192 & 24 & 213 & 25 & 213 & 27 & 186 & 19 \\
\hline & 64 & 182 & 23 & 205 & 24 & 207 & 25 & 190 & 21 \\
\hline
\end{tabular}

TABLE 2. Vectorial reaction-diffusion equation. Number of PCG iteration counts for the divergence-conforming HDG scheme (27).

\begin{tabular}{|c|c|c|c|c|c|c|c|c|c|}
\hline \multirow[t]{2}{*}{$k$} & $N$ & \multicolumn{2}{|c|}{$\tau_{1}=1, \tau_{2}=1$} & \multicolumn{2}{|c|}{$\tau_{1}=1, \tau_{2}=10^{4}$} & \multicolumn{2}{|c|}{$\tau_{1}=10^{4}, \tau_{2}=1$} & \multicolumn{2}{|c|}{$\tau_{1}=10^{4}, \tau_{2}=10^{4}$} \\
\hline & & ASP-ASP & ASP-DIR & ASP-ASP & ASP-DIR & ASP-ASP & ASP-DIR & ASP-ASP & ASP-DIR \\
\hline \multirow{4}{*}{1} & 8 & 16 & 11 & 38 & 25 & 26 & 17 & 12 & 6 \\
\hline & 16 & 16 & 11 & 35 & 24 & 27 & 20 & 13 & 7 \\
\hline & 32 & 16 & 10 & 34 & 23 & 26 & 21 & 14 & 8 \\
\hline & 64 & 15 & 10 & 33 & 23 & 25 & 19 & 16 & 9 \\
\hline \multirow{4}{*}{4} & 8 & 25 & 18 & 39 & 26 & 25 & 19 & 15 & 14 \\
\hline & 16 & 25 & 17 & 40 & 27 & 30 & 21 & 19 & 15 \\
\hline & 32 & 24 & 16 & 39 & 27 & 31 & 22 & 23 & 16 \\
\hline & 64 & 23 & 15 & 38 & 27 & 31 & 22 & 25 & 16 \\
\hline \multirow{4}{*}{7} & 8 & 29 & 22 & 43 & 29 & 27 & 23 & 21 & 20 \\
\hline & 16 & 28 & 20 & 43 & 29 & 33 & 24 & 25 & 20 \\
\hline & 32 & 28 & 19 & 42 & 30 & 34 & 25 & 28 & 19 \\
\hline & 64 & 26 & 18 & 42 & 30 & 33 & 24 & 29 & 19 \\
\hline \multirow{4}{*}{10} & 8 & 34 & 25 & 49 & 34 & 31 & 26 & 26 & 23 \\
\hline & 16 & 33 & 24 & 47 & 34 & 36 & 27 & 30 & 23 \\
\hline & 32 & 33 & 22 & 47 & 33 & 38 & 27 & 33 & 22 \\
\hline & 64 & 31 & 21 & 44 & 32 & 37 & 27 & 34 & 22 \\
\hline
\end{tabular}

TABLE 3. Generalized biharmonic equation, simply supported boundary condition. Number of PCG iteration counts for the CIP-HDG scheme (45).

\section{Conclusion}

We applied the ASP theory to construct robust preconditioners for the HDG schemes for three class of elliptic operators with a low order term. Extension of ASP theory for HDG scheme for 


\begin{tabular}{|c|c|c|c|c|c|c|c|c|c|}
\hline \multirow[t]{2}{*}{$k$} & \multirow[t]{2}{*}{$N$} & \multicolumn{2}{|c|}{$\tau_{1}=1, \tau_{2}=1$} & \multicolumn{2}{|c|}{$\tau_{1}=1, \tau_{2}=10^{4}$} & \multicolumn{2}{|c|}{$\tau_{1}=10^{4}, \tau_{2}=1$} & \multicolumn{2}{|c|}{$\tau_{1}=10^{4}, \tau_{2}=10^{4}$} \\
\hline & & ASP-ASP & ASP-DIR & ASP-ASP & ASP-DIR & ASP-ASP & ASP-DIR & ASP-ASP & ASP-DIR \\
\hline & 8 & 28 & 17 & 36 & 24 & 27 & 18 & 14 & 6 \\
\hline 1 & 16 & 37 & 23 & 39 & 27 & 27 & 20 & 14 & 8 \\
\hline 1 & 32 & 50 & 34 & 48 & 33 & 26 & 20 & 16 & 11 \\
\hline & 64 & 67 & 49 & 62 & 48 & 27 & 23 & 22 & 15 \\
\hline \multirow{4}{*}{4} & 8 & 46 & 28 & 46 & 29 & 25 & 19 & 16 & 14 \\
\hline & 16 & 60 & 41 & 58 & 37 & 30 & 22 & 20 & 17 \\
\hline & 32 & 77 & 53 & 74 & 50 & 33 & 23 & 27 & 20 \\
\hline & 64 & 102 & 74 & 99 & 71 & 40 & 27 & 36 & 23 \\
\hline \multirow{4}{*}{7} & 8 & 53 & 34 & 52 & 33 & 28 & 24 & 21 & 21 \\
\hline & 16 & 69 & 46 & 66 & 42 & 34 & 25 & 24 & 22 \\
\hline & 32 & 90 & 60 & 86 & 58 & 36 & 26 & 32 & 22 \\
\hline & 64 & 117 & 80 & 112 & 79 & 44 & 30 & 42 & 26 \\
\hline \multirow{4}{*}{10} & 8 & 57 & 39 & 54 & 36 & 32 & 27 & 26 & 24 \\
\hline & 16 & 73 & 49 & 70 & 45 & 37 & 28 & 30 & 26 \\
\hline & 32 & 95 & 64 & 91 & 60 & 39 & 29 & 34 & 27 \\
\hline & 64 & 126 & 87 & 119 & 84 & 47 & 31 & 45 & 28 \\
\hline
\end{tabular}

TABLE 4. Generalized biharmonic equation, clamped boundary condition. Number of PCG iteration counts for the CIP-HDG scheme (45).

other elliptic operator is the subject of ongoing research. Robust preconditioning of HDG schemes for saddle point systems is the subject of a forthcoming paper.

\section{REFERENCES}

[1] M. Ainsworth And G. Fu, Fully computable a posteriori error bounds for hybridizable discontinuous Galerkin finite element approximations, J. Sci. Comput., 77 (2018), pp. 443-466.

[2] D. N. ARnold, An interior penalty finite element method with discontinuous elements, SIAM J. Numer. Anal., 19 (1982), pp. 742-760.

[3] B. Ayuso de Dios, F. Brezzi, L. D. Marini, J. Xu, And L. Zikatanov, A simple preconditioner for a discontinuous Galerkin method for the Stokes problem, J. Sci. Comput., 58 (2014), pp. 517-547.

[4] S. C. BRenner AND L.-Y. Sung, $C^{0}$ interior penalty methods for fourth order elliptic boundary value problems on polygonal domains, J. Sci. Comput., 22/23 (2005), pp. 83-118.

[5] L. Chen, J. Wang, Y. WAng, And X. Ye, An auxiliary space multigrid preconditioner for the weak Galerkin method, Comput. Math. Appl., 70 (2015), pp. 330-344.

[6] B. Cockburn, The hybridizable discontinuous Galerkin methods, in Proceedings of the International Congress of Mathematicians. Volume IV, Hindustan Book Agency, New Delhi, 2010, pp. 2749-2775.

[7] B. Cockburn, O. Dubois, J. Gopalakrishnan, and S. Tan, Multigrid for an HDG method, IMA J. Numer. Anal., 34 (2014), pp. 1386-1425.

[8] B. Cockburn, J. Gopalakrishnan, and R. Lazarov, Unified hybridization of discontinuous Galerkin, mixed and continuous Galerkin methods for second order elliptic problems, SIAM J. Numer. Anal., 47 (2009), pp. 13191365.

[9] B. Cockburn, N. C. Nguyen, and J. Peraire, HDG methods for hyperbolic problems, in Handbook of numerical methods for hyperbolic problems, vol. 17 of Handb. Numer. Anal., Elsevier/North-Holland, Amsterdam, 2016, pp. 173-197.

[10] R. D. FAlgout And U. M. YAng, HYPRE: a library of high performance perconditioners, In Preconditiners, Lecture Notes in Computer Science, pp. 632-641, 2002.

[11] V. E. Henson And U. M. YAng, BoomerAMG: a parallel algebraic multigrid solver and preconditioner, vol. 41, 2002, pp. 155-177. Developments and trends in iterative methods for large systems of equations-in memoriam Rüdiger Weiss (Lausanne, 2000). 
[12] G. Kanschat And N. Sharma, Divergence-conforming discontinuous Galerkin methods and $C^{0}$ interior penalty methods, SIAM J. Numer. Anal., 52 (2014), pp. 1822-1842.

[13] C. Lehrenfeld, Hybrid Discontinuous Galerkin methods for solving incompressible flow problems. Diploma Thesis, MathCCES/IGPM, RWTH Aachen, 2010.

[14] B. LI AND X. XIE, BPX preconditioner for nonstandard finite element methods for diffusion problems, SIAM J. Numer. Anal., 54 (2016), pp. 1147-1168.

[15] S. Muralikrishnan, T. Bui-Thanh, And J. N. Shadid, A multilevel approach for trace system in HDG discretizations, J. Comput. Phys., 407 (2020), pp. 109240, 26.

[16] S. V. Nepomnyaschikh, Mesh theorems on traces, normalizations of function traces and their inversion, Soviet J. Numer. Anal. Math. Modelling, 6 (1991), pp. 223-242.

[17] NGSoLve, Finite element software, www.ngsolve.org.

[18] N. C. NGuyen And J. Peraire, Hybridizable discontinuous Galerkin methods for partial differential equations in continuum mechanics, J. Comput. Phys., 231 (2012), pp. 5955-5988.

[19] N. C. Nguyen, J. Peraire, and B. Cockburn, Hybridizable discontinuous Galerkin methods, in Spectral and high order methods for partial differential equations, vol. 76 of Lect. Notes Comput. Sci. Eng., Springer, Heidelberg, 2011, pp. 63-84.

[20] J. SchöBERL, C++11 Implementation of Finite Elements in NGSolve, 2014. ASC Report 30/2014, Institute for Analysis and Scientific Computing, Vienna University of Technology.

[21] J. XU, The auxiliary space method and optimal multigrid preconditioning techniques for unstructured grids, vol. 56, 1996, pp. 215-235. International GAMM-Workshop on Multi-level Methods (Meisdorf, 1994).

[22] — Fast Poisson-based solvers for linear and nonlinear PDEs, in Proceedings of the International Congress of Mathematicians. Volume IV, Hindustan Book Agency, New Delhi, 2010, pp. 2886-2912.

[23] Z. ZAGLmAYR, High Order Finite Element Methods for Electromagnetic Field Computation, 2006. PhD thesis, Johannes Kepler Universität Linz, Linz.

[24] L. T. Zikatanov, Two-sided bounds on the convergence rate of two-level methods, Numer. Linear Algebra Appl., 15 (2008), pp. 439-454. USA.

Department of Applied and Computational Mathematics and Statistics, University of Notre Dame, Email address: gfu@nd.edu 